\title{
Odd Inverse Power Generalized Weibull Generated Family of Distributions: Properties and Applications
}

\author{
A.S. Al-Moisheer $\mathbb{D}^{\mathbb{D}},{ }^{1}$ I. Elbatal, ${ }^{2}$ Waleed Almutiry $\mathbb{D}^{3},{ }^{3}$ and Mohammed Elgarhy $\mathbb{D}^{4}$ \\ ${ }^{1}$ Department of Mathematics, College of Science, Jouf University, P. O. Box 848, Sakaka 72351, Saudi Arabia \\ ${ }^{2}$ Department of Mathematics and Statistics, College of Science, Imam Mohammad Ibn Saud Islamic University (IMSIU), \\ Riyadh, Saudi Arabia \\ ${ }^{3}$ Department of Mathematics, College of Science and Arts, Qassim University, Ar Rass, Saudi Arabia \\ ${ }^{4}$ The Higher Institute of Commercial Sciences, Al Mahalla Al Kubra, Algarbia 31951, Egypt
}

Correspondence should be addressed to Mohammed Elgarhy; m_elgarhy85@sva.edu.eg

Received 5 September 2021; Revised 4 October 2021; Accepted 18 October 2021; Published 9 November 2021

Academic Editor: Lazim Abdullah

Copyright (c) 2021 A.S. Al-Moisheer et al. This is an open access article distributed under the Creative Commons Attribution License, which permits unrestricted use, distribution, and reproduction in any medium, provided the original work is properly cited.

\begin{abstract}
A novel family of produced distributions, odd inverse power generalized Weibull generated distributions, is introduced. Various mathematics structural properties for the odd inverse power generalized Weibull generated family are computed. Numerical analysis for mean, variance, skewness, and kurtosis is performed. The new family contains many new models, and the densities of the new models can be right skewed and symmetric with "unimodal" and "bimodal" shapes. Also, its hazard rate function can be "constant," "decreasing," "increasing," "increasing-constant," "upside-down-constant," and "decreasing-constant." Different types of entropies are calculated. Some numerical values of various entropies for some selected values of parameters for the odd inverse power generalized Weibull exponential model are computed. The maximum likelihood estimation, least square estimation, and weighted least square estimation approaches are used to estimate the OIPGW-G parameters. Many bivariate and multivariate type models have been also derived. Two real-world data sets are used to demonstrate the new family's use and versatility.
\end{abstract}

\section{Introduction}

There are many applications for inverse (I) distributions, including econometrics, biology, life testing, engineering sciences as well as medical investigation and survey sampling concerns. It is also used in financial literature, environmental studies, survival and dependability theory, and other fields. By applying the inverse transformation to well-known random variables that display distinct properties of density and hazard rate shape, several writers have explored (lifetime) phenomena that a noninverted distribution cannot examine, for example, the I exponential model by [1], I Rayleigh distribution by [2], I Lindley distribution by [3], I power Lindley distribution by [4], I Kumaraswamy distribution by [5], Nadarajah-Haghighi distribution by [6], and I Topp Leone by [7], among others.
The author in [8] introduced a new three-parameter distribution called I power generalized Weibull distribution. The distribution function (CDF) of I power generalized Weibull (IPGW) distribution is given by

$$
G_{\lambda, \alpha, \theta}(z)=\exp \left[1-\left(1+\theta z^{-\alpha}\right)^{\lambda}\right], \quad z>0,
$$

where $\theta>0$ is a scale parameter and $\alpha, \lambda>0$ are shape parameters. The associated density function (PDF) is as follows:

$$
g_{\lambda, \alpha, \theta}(z)=\theta \alpha \lambda z^{-\alpha-1}\left(1+\theta z^{-\alpha}\right)^{\lambda-1} \exp \left[1-\left(1+\theta z^{-\alpha}\right)^{\lambda}\right] .
$$

Recently, statisticians have been interested in proposing new families of univariate distributions that are derived from 
existing ones. Adding one or more form parameters results in these new generators, which improve accuracy and flexibility in modeling for a variety of diverse real-life applications. The most recent families of distributions to appear in the literature are as follows: a method for introducing a parameter into a family of distributions by [9], beta- $G$ by [10], odd Nadarajah-Haghighi-G by [11], the odd Lindley- $G$ by [12], and the odd Fréchet- $G$ by [13], odd generalized exponential- $G$ by [14], exponentiated power generalized Weibull power series family of distributions by [15], and odd generalized $\mathrm{NH}-\mathrm{G}$ by [16], among others.

Using [17] T-X concept, we create a new broader and more flexible family of distributions known as the odd I power generalized Weibull-G (OIPGW-G) family.

$$
F_{\underline{\mathbf{V}}}(z)=\left.\exp \left\{1-\left[1+\theta \mathbf{O}_{\underline{\xi}}^{\alpha}(z)\right]^{\lambda}\right\}\right|_{\underline{\mathbf{V}}=\lambda, \alpha, \theta, \underline{\xi}>0 \text { and } z \in \mathbb{R}},
$$

where $\mathbf{O}_{\xi}^{\alpha}(z)=(\bar{G}(z ; \underline{\xi}) / G(z ; \underline{\xi}))^{\alpha}$. The corresponding PDF is given by

$$
f_{\underline{\mathbf{v}}}(z)=\theta \alpha \lambda \frac{g_{\underline{\xi}}(z) \bar{G}_{\underline{\xi}}(z)^{\alpha-1}\left[1+\theta \mathbf{O}_{\underline{\xi}}^{\alpha}(z)\right]^{\lambda-1}}{G_{\underline{\xi}}(z)^{\alpha+1} \exp \left\{\left[1+\theta \mathbf{O}_{\underline{\xi}}^{\alpha}(z)\right]^{\lambda}-1\right\}} .
$$

A random variable (RV) $Z$ with $\mathrm{PDF}$ (4) is now indicated as $Z \sim \mathrm{OIPGW}-\mathrm{G}(\underline{\mathrm{V}})$. The reliability function $(\mathrm{RF})$ and hazard rate function (HRF) can be derived from each other as $\bar{F}_{\underline{\mathbf{V}}}(z)=1-F_{\underline{\mathbf{V}}}(z)$ and $\tau(z ; \underline{\mathbf{V}})=f_{\underline{\mathbf{V}}}(z) / \bar{F}_{\underline{\mathbf{V}}}(z)$. The following is an interpretation of the OIPG $\overline{\mathrm{W}}-\mathrm{G}$ family. Let $Y$ be a RV with a continuous $G$ distribution that describes a stochastic system. The probability that a person (or component) will not be working (failure or death) at time $z$ after a lifespan of $Y$ is characterized with $(G(z ; \xi) / \bar{G}(z ; \xi))$. The $\mathrm{RV} Z$ represents the variability of this chances of failure, and we suppose that it follows the OIPGW-G family of parameters $\theta, \alpha$, and $\lambda$, and the $\operatorname{CDF}$ of $Z$ it is possible to write

$$
P(Y \leq z)=\operatorname{Pr}\left(Z \leq \frac{G_{\underline{\xi}}(z)}{\bar{G}_{\underline{\xi}}(z)}\right)=F_{\underline{\mathbf{V}}}(z) .
$$

As a result of this, OIPGW-G family is used for the following reasons:

(i) Specific models with all sorts of HRFs to be defined

(ii) Better matches than other models with the same baseline distribution that has been generated

(iii) Make kurtosis more flexible than the baseline model

(iv) The pdf can be symmetric or right or left skewed and reversed J shaped. An extremely versatile model, the OIPGW-G family of distributions is able to adapt to a variety of various models when its parameters are altered. The OIPGW-G family of distributions includes the following well-known families as special cases in Table 1.

The quantile function $(\mathrm{QF}) Q_{G}(u)$ is given by the relation as follows:
TABLE 1: Submodels of the OIPGW-G family.

\begin{tabular}{ccccc}
\hline$\lambda$ & $\theta$ & $\alpha$ & Reduced family & Authors \\
\hline 1 & - & - & Odd Fréchet-G & Haq and Elgarhy \\
& & Inverse Nadarajah-Haghighi- & (2018) \\
- & - & 1 & $\mathrm{New}$ \\
1 & - & 1 & Inverse exponential-G family & $\mathrm{New}$ \\
1 & -2 & Inverse Rayleigh- $G$ & $\mathrm{New}$ \\
\hline
\end{tabular}

$$
F^{-1}(u)=Q_{G}(u)=G^{-1}\left(1+\left\{\frac{1}{\theta}\left[(1-\ln u)^{(1 / \lambda)}-1\right]\right\}^{(1 / \alpha)}\right)^{-1} .
$$

Equation (6) can be used in deriving the Bowley skewness coefficient and the Moors kurtosis coefficient.

The following is how the rest of this article is organized. The linear form of the PDF and CDF for the new family is expressed in Section 2. Section 3 contains several special models of the OIPGW-G family. Section 4 discusses the new family's structural characteristics as moments (Mos), incomplete Mos (IMos), mean deviations (MDes), Bonferroni (Bon) and Lorenz (Lor) curves, moment generating function (MoGF), and Probability Weighted Moments (PrWMos). Also, some numerical analyses for the mean $\mathrm{M}(\mathrm{Z})$, variance $\operatorname{Var}(Z)$, coefficient of skewness CS(Z), coefficient of kurtosis $\mathrm{CK}(\mathrm{Z})$, and coefficient of variation $\mathrm{CV}(\mathrm{Z})$ are discussed in the same section. Section 5 computes entropies of various sorts and also some numerical values of different entropies for specific selected parameter values for the odd inverse power generalized Weibull exponential model. In Section 6, parameters are estimated using three different techniques as the maximum likelihood (ML), least square (LS), and weighted LS (WLS) techniques. Many bivariate and multivariate type models have also been examined in Section 7. In Section 8, two applications to real-world data sets illustrate the empirical significance of the odd inverse power generalized Weibull exponential model. At the end of the paper, there are conclusions.

\section{Important Representation}

We give a helpful linear form for the OIPGW-G PDF in this section. If $\left|z_{1} / z_{2}\right|<1$ and $\nabla>0$ is a real noninteger, then the next power series (PS) expansions hold.

$$
\begin{aligned}
& \left(1-\frac{z_{1}}{z_{2}}\right)^{-\nabla}=\sum_{\kappa=0}^{\infty} \frac{\Gamma(\nabla+\kappa)}{\kappa ! \Gamma(\nabla)}\left(\frac{z_{1}}{z_{2}}\right)^{\kappa}, \\
& \left(1-\frac{z_{1}}{z_{2}}\right)^{\nabla}=\sum_{\kappa=0}^{\infty} \frac{(-1)^{\kappa} \Gamma(1+\nabla)}{\kappa ! \Gamma(1+\nabla-\kappa)}\left(\frac{z_{1}}{z_{2}}\right)^{\kappa},
\end{aligned}
$$

and an exponential function is implemented by using a PS, and we get

$$
\exp \left\{-\left[1+\theta \mathbf{O}_{\underline{\xi}}^{\alpha}(z)\right]^{\lambda}\right\}=\sum_{\hbar=0}^{\infty} \frac{1}{\hbar !}(-1)^{\hbar}\left[1+\theta \mathbf{O}_{\underline{\xi}}^{\alpha}(z)\right]^{\lambda \hbar} .
$$


Inserting (9) in (4), we have

$$
f_{\underline{\mathbf{V}}}(z)=\theta \alpha \lambda \exp (1) g_{\underline{\xi}}(z) \sum_{\hbar=0}^{\infty} \frac{(-1)^{\hbar}}{\hbar !} \frac{\bar{G}_{\underline{\xi}}(z)^{\alpha-1}}{G_{\underline{\xi}}(z)^{\alpha+1}} \underbrace{\left[1+\theta \mathbf{O}_{\underline{\xi}}^{\alpha}(z)\right]^{\lambda(\hbar+1)-1}}_{A(z)} .
$$

Using (8), we can write inserting (11) into (10). Then, equation (10) can be written as

$$
A(z)=\left.\sum_{\hbar=0}^{\infty} \frac{\theta^{w} \Gamma(\lambda)}{w ! \Gamma\left(\lambda^{\cdot}-w\right)} \frac{\bar{G}_{\underline{\xi}}(z)^{\alpha w}}{G_{\underline{\xi}}(z)^{\alpha w}}\right|_{\lambda=\lambda(\hbar+1)},
$$

$$
f_{\underline{\mathbf{V}}}(z)=\left.\theta \alpha \lambda \exp (1) g_{\underline{\xi}}(z) \sum_{\hbar, w=0}^{\infty} \frac{(-1)^{\hbar} \theta^{w} \Gamma(\lambda)}{\hbar ! w ! \Gamma(\lambda-w)} \frac{\bar{G}_{\underline{\xi}}(z)^{\alpha-1}}{G_{\underline{\xi}}(z)^{\alpha+1}}\right|_{\alpha=\alpha(w+1)} .
$$

Applying (7) to $G_{\xi}(z)^{\alpha+1}$, we can write

$$
G_{\underline{\xi}}(z)^{-(\alpha+1)}=\sum_{\kappa=0}^{\infty} \frac{\Gamma(\alpha+\kappa+1)}{\kappa ! \Gamma(\alpha+1)}\left[1-G_{\underline{\xi}}(z)\right]^{\kappa} .
$$

$$
f_{\underline{\mathbf{V}}}(z)=\sum_{m=0}^{\infty} \Delta_{m} \pi_{m+1}(z)
$$

where

Substituting (13) into (12) and applying (8), the OIPGW$\mathrm{G} P D F$ is

$$
\Delta_{m}=\sum_{\hbar, w, \kappa=0}^{\infty} \frac{(-1)^{\hbar} \theta^{w+1} \Gamma(\lambda) \Gamma(\alpha+\kappa+1) \Gamma(\alpha+\kappa)}{\hbar ! w ! \kappa ! m !(m+1) \Gamma(\lambda(\hbar+1)-w) \Gamma(\alpha+1) \Gamma(\alpha+\kappa-m)}
$$

and $\pi_{m+1}(z)=(m+1) g(z) G^{m}(z)$ is the exp-G PDF with power parameter $(m+1)$. Also, the CDF of the OIPGW-G family is

$$
F_{\underline{\mathbf{V}}}(z)=\sum_{m=0}^{\infty} \Delta_{m} \Pi_{m+1}(z),
$$

where $\Pi_{m+1}(z)$ is the exp-G CDF with power parameter $m+1$.

\section{Some Special Models of the OIPGW-G Family}

In this part, we described four submodels of the OIPGW-G family of distributions. There is no doubt about it, and the PDF (4) will be the most tractable when the CDF $G(z)$ and PDF $g(z)$ have easy-to-understand analytic expressions. When we start with the baseline distributions: uniform (U), exponential (Ex), Weibull (W), and Rayleigh (R), we get at four submodels of this family. Table 2 shows the CDF and PDF of various baseline models.

Figures 1 and 2 represent the plots of PDFs and the HRFs for the models which are reported in Table 2. From Figure 1, we can note that the PDFs can be right skewed and symmetric with "unimodal" and "bimodal" shapes. The HRFs can be "constant," "decreasing," "increasing," "increasing- constant," "upside-down-constant," and "decreasingconstant."

\section{Statistical Properties}

4.1. Ordinary Moments and Incomplete Moments Functions. The $r^{\text {th }}$ ordinary Mos of $Z$, say $\mu_{r}^{\prime}$, is driven from (14) as

$$
\mu_{r}^{\prime}=E\left(Z^{r}\right)=\sum_{m=0}^{\infty} \Delta_{m} Z_{m+1}^{r}(z),
$$

where $Z_{m+1}$ has a power parameter of $(m+1)$ and represents the exp-G random variable. It is possible to obtain the CS and CK measures by using the $n^{\text {th }}$ central Mos, say $M_{n}(z)$ of $Z$, where

$$
\begin{aligned}
M_{n}(z) & =E\left(Z-\mu_{1}^{\prime}\right)^{n}=\sum_{r=0}^{n}\left(\begin{array}{c}
n \\
r
\end{array}\right)\left(-\mu_{1}^{\prime}\right)^{n-r} E\left(Z^{r}\right) \\
& =\sum_{r=0}^{n} \sum_{\kappa, m=0}^{\infty}\left(\begin{array}{l}
n \\
r
\end{array}\right)\left(-\mu_{1}^{\prime}\right)^{n-r} \eta_{\kappa, m} E\left(Z_{m+1}^{r}\right) .
\end{aligned}
$$

Also, the cumulants $\left(\varkappa_{s}\right)$ of $Z$ follow recursively from 
TABLE 2: The CDF and PDF of these baseline models.

\begin{tabular}{lccc}
\hline Model & $G_{\underline{\xi}}(z)$ & $\mathbf{O}_{\xi}^{\alpha}(z)$ & $\mathrm{CDF}$ \\
\hline Uniform & $(z / \beta)$ & $((\beta / z)-1)^{\alpha}$ & $\exp \left\{1-\left[1+\theta((\beta / z)-1)^{\alpha}\right]^{\lambda}\right\}$ \\
Exponential & $1-\exp (-\beta z)$ & {$[\exp (\beta z)-1]^{-\alpha}$} & $\exp \left(1-\left\{1+\theta[\exp (\beta z)-1]^{-\alpha}\right\}^{\lambda}\right)$ \\
Weibull & $1-\exp \left(-z^{\beta}\right)$ & {$\left[\exp \left(z^{\beta}\right)-1\right]^{-\alpha}$} & $\exp \left(1-\left\{1+\theta\left[\exp \left(z^{\beta}\right)-1\right]^{-\alpha}\right\}^{\lambda}\right)$ \\
Rayleigh & $1-\exp \left(-\beta z^{2}\right)$ & {$\left[\exp \left(z^{2}\right)-1\right]^{-\alpha}$} & $\exp \left(1-\left\{1+\theta\left[\exp \left(\beta z^{2}\right)-1\right]^{-\alpha}\right\}^{\lambda}\right)$ \\
\hline
\end{tabular}
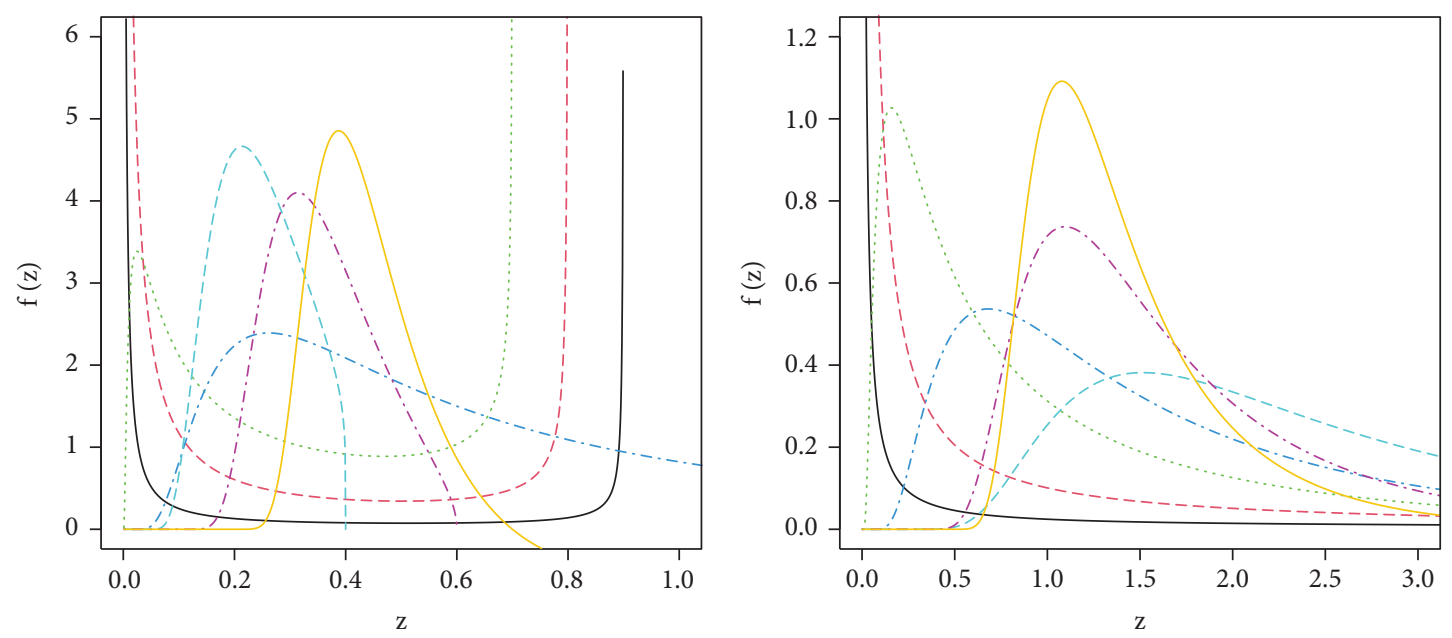

$\begin{aligned} \alpha & =0.2 \theta=0.4 \lambda=0.3 \beta=0.9 \\ --\alpha & =0.4 \theta=0.6 \lambda=0.5 \beta=0.8 \\ \alpha & =0.6 \theta=0.8 \lambda=0.7 \beta=0.7 \\ --\alpha=1.0 \theta & =1.0 \lambda=0.9 \beta=0.6 \\ --\alpha & =1.2 \theta=0.9 \lambda=1.2 \beta=0.4 \\ -\alpha & =1.5 \theta=0.7 \lambda=1.5 \beta=0.6 \\ \alpha & =2.0 \theta=0.8 \lambda=1.7 \beta=0.7\end{aligned}$

$\alpha=0.2 \theta=0.4 \lambda=0.3 \beta=0.9$

$--\alpha=0.4 \theta=0.6 \lambda=0.5 \beta=0.8$

$\alpha=0.8 \theta=0.8 \lambda=0.7 \beta=0.7$

$\ldots \alpha=1.0 \theta=1.0 \lambda=0.9 \beta=0.6$

$--\alpha=1.2 \theta=0.9 \lambda=1.2 \beta=0.4$

-. $\alpha=1.5 \theta=0.7 \lambda=1.5 \beta=0.6$

$\alpha=2.0 \theta=0.8 \lambda=1.7 \beta=0.7$
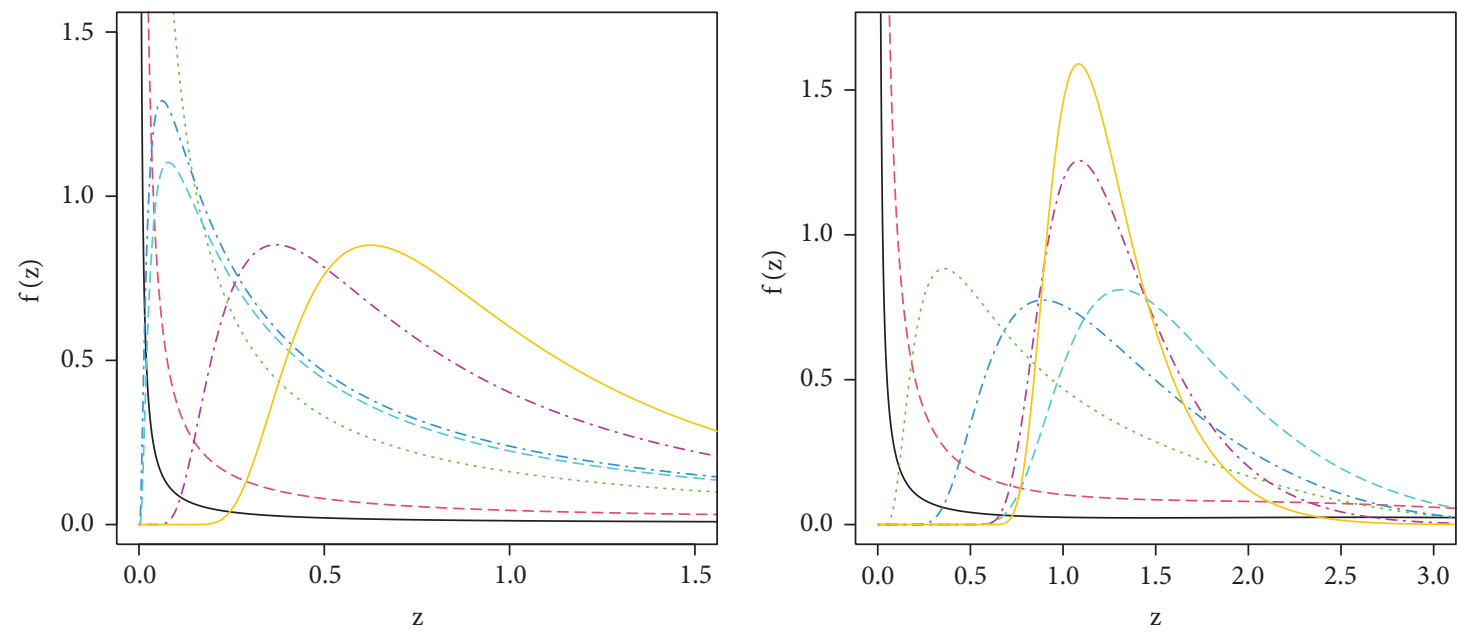

$\begin{aligned} & \alpha=0.1 \theta=0.4 \lambda=0.3 \beta=0.9 \\ &--\alpha=0.2 \theta=0.6 \lambda=0.5 \beta=0.8 \\ & \cdots \alpha=0.6 \theta=0.8 \lambda=0.7 \beta=0.7 \\ &-\alpha=0.8 \theta=1.0 \lambda=0.9 \beta=0.6 \\ &--\alpha=1.0 \theta=0.9 \lambda=1.2 \beta=0.4 \\ & \alpha=1.2 \theta=0.7 \lambda=1.5 \beta=0.6 \\ & \alpha=1.4 \theta=0.8 \lambda=1.7 \beta=0.7\end{aligned}$

$\begin{aligned} &-\alpha=0.1 \theta=0.4 \lambda=0.3 \beta=0.9 \\ &--\alpha=0.2 \theta=0.6 \lambda=0.5 \beta=0.8 \\ & \cdots \alpha=0.6 \theta=0.8 \lambda=0.7 \beta=0.7 \\ & \alpha \alpha=0.8 \theta=1.0 \lambda=0.9 \beta=0.6 \\ &--\alpha=1.0 \theta=0.9 \lambda=1.2 \beta=0.4 \\ &--\alpha=1.2 \theta=0.7 \lambda=1.5 \beta=0.6 \\ & \alpha=1.4 \theta=0.8 \lambda=1.7 \beta=0.7\end{aligned}$

FIgure 1: The plots of PDFs for OIPGWU, OIPGWEx, OIPGWW, and OIPGWR distributions. 

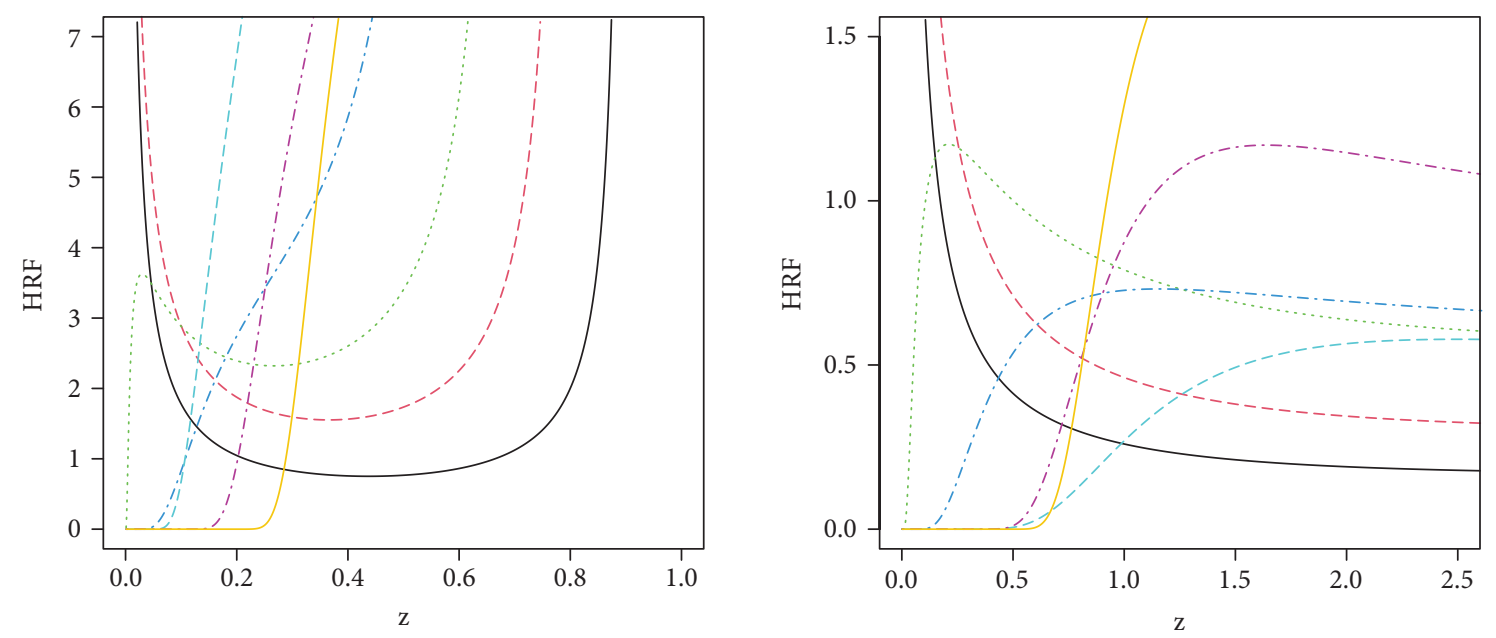

$\begin{aligned} &-\alpha=0.2 \theta=0.4 \lambda=0.3 \beta=0.9 \\ &--\alpha=0.4 \theta=0.6 \lambda=0.5 \beta=0.8 \\ & \cdots \alpha=0.6 \theta=0.8 \lambda=0.7 \beta=0.7 \\ &-\alpha=1.0 \theta=1.0 \lambda=0.9 \beta=0.6 \\ &-\alpha=1.2 \theta=0.9 \lambda=1.2 \beta=0.4 \\ & \alpha=1.5 \theta=0.7 \lambda=1.5 \beta=0.6 \\ & \alpha=2.0 \theta=0.8 \lambda=1.7 \beta=0.7\end{aligned}$
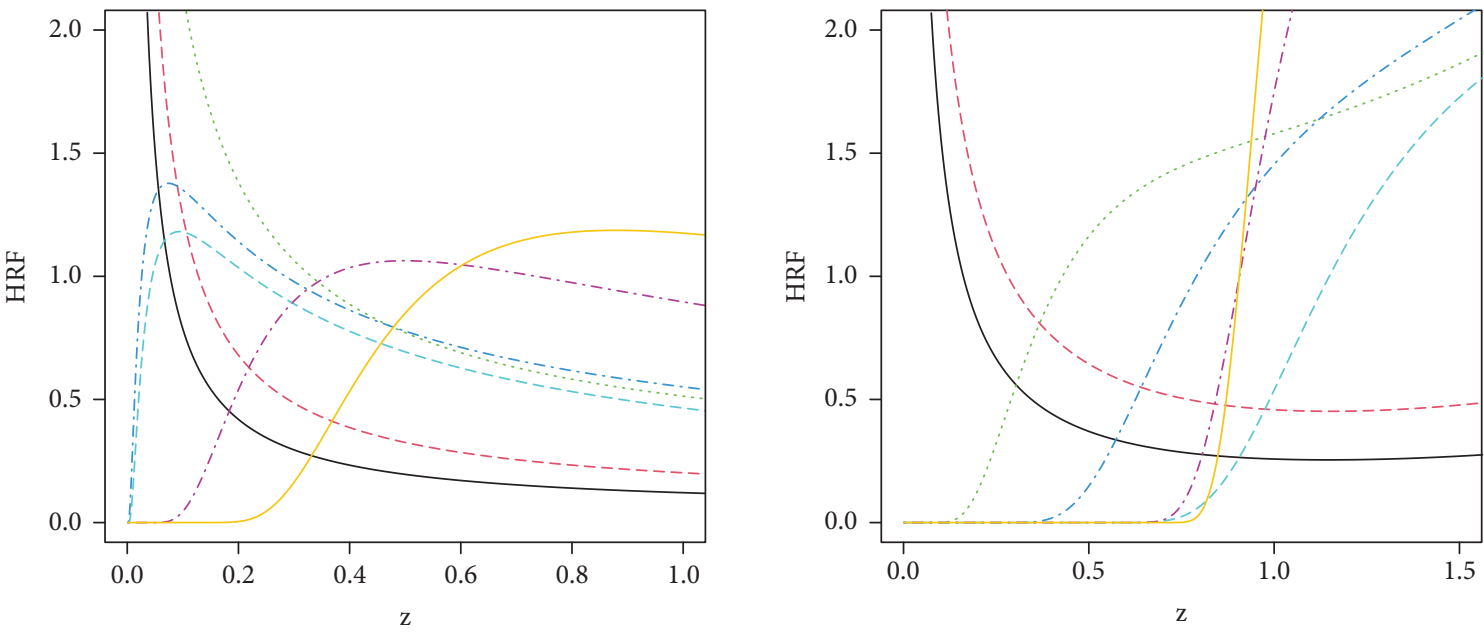

$\begin{aligned} &-\alpha=0.1 \theta=0.4 \lambda=0.3 \beta=0.9 \\ &--\alpha=0.2 \theta=0.6 \lambda=0.5 \beta=0.8 \\ & \alpha \alpha=0.6 \theta=0.8 \lambda=0.7 \beta=0.7 \\ &--\alpha=0.8 \theta=1.0 \lambda=0.9 \beta=0.6 \\ &--\alpha=1.0 \theta=0.9 \lambda=1.2 \beta=0.4 \\ &--\alpha=1.2 \theta=0.7 \lambda=1.5 \beta=0.6 \\ & \alpha=1.4 \theta=0.8 \lambda=1.7 \beta=0.7\end{aligned}$

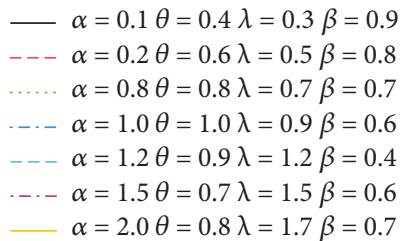

FIgURE 2: The plots of HRFs for OIPGWU, OIPGWEx, OIPGWW, and OIPGWR distributions.

$$
\varkappa_{s}=\mu_{s}^{\prime}-\sum_{d=0}^{s-1}\left(\begin{array}{c}
s-1 \\
d-1
\end{array}\right) \varkappa_{d} \mu_{s-d}^{\prime},
$$

where $\varkappa_{1}=\mu_{1}^{\prime}, \varkappa_{2}=\mu_{2}^{\prime}-\mu_{1}^{\prime 2}$, and $\varkappa_{3}=\mu_{3}^{\prime}-3 \mu_{2}^{\prime} \mu_{1}^{\prime}+\mu_{1}^{\prime 3}$.

It is possible to determine the MDes, Bon, and Lor curves using the first IMos. It is highly important in economics and dependability as well as in demography as well as in the fields of insurance and medical. Not only in econometrics but also in many other fields as well, this is apparent. The $s^{\text {th }}$ IMos of
$Z$ defined by $v_{s}(t)$ for any real $s>0$ can be investigated from (14) as

$$
v_{s}(t)=\int_{-\infty}^{t} z^{s} f(z) \mathrm{d} z=\sum_{m=0}^{\infty} \Delta_{m} \int_{-\infty}^{t} z^{s} \pi_{m+1}(z) \mathrm{d} z .
$$

Equation (20) denotes the $s^{\text {th }}$ incomplete moments of $Z_{m+1}$. As well as providing significant information on population characteristics, the MDes have been used to income fields and property in the field of economics for a 
long time. If $Z$ has the OIPGW-G family of distribution, then the MDes about the mean $\mu=E(Z)$ and the MDes about the median $M$ are defined by

$$
\begin{aligned}
D_{\mu}(z) & =E\left|Z-\mu_{1}^{\prime}\right|=2 \mu_{1}^{\prime} F\left(\mu_{1}^{\prime}\right)-2 v_{1}\left(\mu_{1}^{\prime}\right), \\
D_{M}(z) & =E|Z-M|=\mu_{1}^{\prime}-2 v_{1}(M),
\end{aligned}
$$

respectively, where $\mu_{1}^{\prime}=E(z), M=\operatorname{median}(z)=Q(1 / 2)$, $F\left(\mu_{1}^{\prime}\right)$ is evaluated from (3), and $v_{1}(t)$ is the first IMo given by (20) with $s=1$, where

$$
v_{1}(t)=\int_{-\infty}^{t} z f(z) \mathrm{d} z=\sum_{m=0}^{\infty} \Delta_{m} \int_{-\infty}^{t} z \pi_{m+1}(z) \mathrm{d} z .
$$

We can determine $\delta_{\mu}(z)$ and $\delta_{M}(z)$ by two techniques; the first can be obtained from (14) as $v_{1}(t)=\sum_{m=0}^{\infty} \Delta_{m} Z_{m+1}(t)$ where $Z_{m+1}(t)=\int_{-\infty}^{t} z \pi_{m+1}(z) \mathrm{d} z$ is the first IMo of the exp- $G$ distribution. The second technique is given by $v_{1}(t)=\sum_{m=0}^{\infty} \Delta_{m} \gamma_{m+1}(t)$ where

$$
\gamma_{m+1}(t)=(m+1) \int_{0}^{G(t)} u^{m+1} Q_{G}(u) \mathrm{d} u,
$$

can be calculated numerically and $Q_{G}(u)=G^{-1}(u ; \varphi)$. The Lor and Bon curves, for a given probability $p$, are given by $L(p)=\left(1 / \mu_{1}^{\prime}\right) v_{1}(q)$ and $B(p)=\left(1 / p \mu_{1}^{\prime}\right) v_{1}(q)$, respectively, where $\mu_{1}^{\prime}=E(z)$, and $q=Q(p)$ is the QF of $Z$ at $p$. Tables 3 and 4 give numerical analysis for $\mathrm{M}(\mathrm{Z}), \operatorname{Var}(\mathrm{Z}), \mathrm{CS}(\mathrm{Z})$, $\mathrm{CK}(\mathrm{Z})$, and $\mathrm{CV}(\mathrm{Z})$.

Figures 3-6 represent 3D plots of $M(Z), \operatorname{Var}(Z), \operatorname{CS}(Z)$, and CK $(Z)$ of the OIPWU, OIPWEx, OIPWW, and OIPWR distributions for several values of parameters.

From Figures 3-6, $M(Z)$ and $\operatorname{Var}(Z)$ are decreasing for all models, $\operatorname{CS}(Z)$ can be can be negative or positive, indicating left or right skewed, and $\mathrm{CK}(Z)$ can be increasing.

The first formula of MoGF may be computed as follows from equation (14):

$$
M_{Z}(t)=E\left(e^{t Z}\right)=\sum_{m=0}^{\infty} \Delta_{m} M_{m+1}(t)
$$

TABLe 3: Numerical values of $M(Z), \operatorname{Var}(Z), \operatorname{CS}(Z), \mathrm{CK}(Z)$, and $\mathrm{CV}(Z)$ at $\theta=\beta=\alpha=0.5$ for the OIPGWEx model.

\begin{tabular}{lccccc}
\hline$\lambda$ & $M(Z)$ & $\operatorname{Var}(Z)$ & $\mathrm{CS}(Z)$ & $\mathrm{CK}(Z)$ & $\mathrm{CV}(Z)$ \\
\hline 0.2 & 0.472 & 3.003 & 6.466 & 60.013 & 3.669 \\
0.5 & 1.164 & 6.699 & 4.039 & 25.403 & 2.224 \\
0.8 & 1.817 & 9.544 & 3.184 & 17.05 & 1.7 \\
1.2 & 2.616 & 12.306 & 2.618 & 12.656 & 1.341 \\
1.5 & 3.16 & 13.803 & 2.37 & 11.019 & 1.176 \\
2.0 & 3.971 & 15.545 & 2.113 & 9.518 & 0.993 \\
\hline
\end{tabular}

TABLe 4: Numerical values of $M(Z), \operatorname{Var}(Z), \operatorname{CS}(Z), \operatorname{CK}(Z)$, and $\mathrm{CV}(Z)$ at $\theta=\beta=\lambda=0.5$ for the OIPGWEx model.

\begin{tabular}{lccccc}
\hline$\alpha$ & $M(Z)$ & $\operatorname{Var}(Z)$ & $\mathrm{CS}(Z)$ & $\mathrm{CK}(Z)$ & $\mathrm{CV}(Z)$ \\
\hline 0.3 & 1.653 & 18.605 & 4.19 & 26.404 & 2.609 \\
0.8 & 0.976 & 2.655 & 3.686 & 22.76 & 1.67 \\
1.2 & 0.95 & 1.219 & 3.172 & 18.866 & 1.162 \\
1.5 & 0.97 & 0.8 & 2.837 & 16.383 & 0.922 \\
2.0 & 1.016 & 0.468 & 2.399 & 13.292 & 0.673 \\
\hline
\end{tabular}

where $M_{m+1}(t)$ is the MoGF of $Z_{m+1}$. Consequently, $M_{Z}(t)$ can be easily determined from the exp- $G$ MoGF. A second alternative formula can be computed from (14) as $M_{Z}(t)=$ $\sum_{m=0}^{\infty} \Delta_{m} \delta(t, m+1)$ where $\delta(t, \varphi)=\varphi \int_{0}^{1} u^{\varphi-1} e^{t Q_{G}(u)} \mathrm{d} u$.

4.2. Probability Weighted Moments. The $(s, r)^{\text {th }}$ PrWMos of the OIPGW-G family is

$$
\Upsilon_{(s, r)}=E\left[Z^{r} F(z)^{r}\right]=\int_{-\infty}^{\infty} z^{s} F(z)^{r} f(z) \mathrm{d} z,
$$

from (3) and (4), and after some algebra, we get

$$
f(z) F(z)^{r}=\sum_{m=0}^{\infty} b_{m} \pi_{m+1}(z),
$$

where

$$
\begin{aligned}
b_{m}= & \exp (1+r) \alpha \lambda \sum_{\hbar, w, \kappa=0}^{\infty} \frac{\theta^{w+1}(r+1)^{\hbar}(-1)^{\hbar+m} \Gamma(\lambda(\hbar+1)) \Gamma(\alpha+\kappa-1)}{w ! \kappa ! m ! \Gamma(r+2) \Gamma(\lambda(\hbar+1)-w) \Gamma\left(\alpha^{\prime}-1\right)} \\
& \times \frac{\Gamma(\alpha+\kappa)}{\Gamma(\alpha+\kappa-m))(m+1)} .
\end{aligned}
$$

Therefore, the $(s, r)^{\text {th }}$ PrWMos of the OIPGW-G family can be expressed as

$$
\Upsilon_{(s, r)}=\sum_{m=0}^{\infty} b_{m} \int_{-\infty}^{\infty} z^{s} \pi_{m+1}(z) \mathrm{d} z,
$$

Thus, the $(s, r)^{\text {th }}$ PrWMos of $Z$ is

$$
\Upsilon_{(s, r)}=\sum_{m=0}^{\infty} b_{m} E\left(Z_{m+1}^{s}\right) .
$$

\section{Entropies}

This section is dedicated to obtain the expression for different entropy measures of the OIPGW-G family. The Rényi entropy (RéE), presented by [18], is defined by 


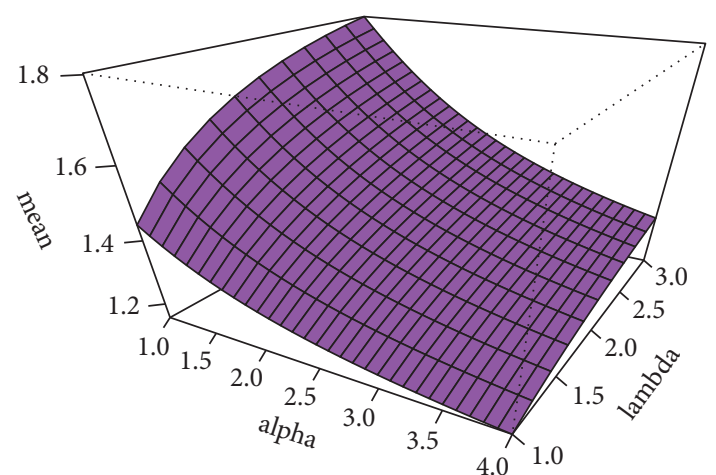

mean

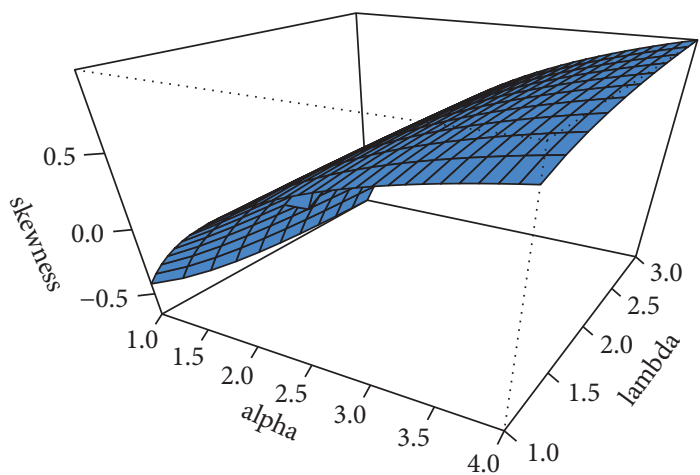

skewness

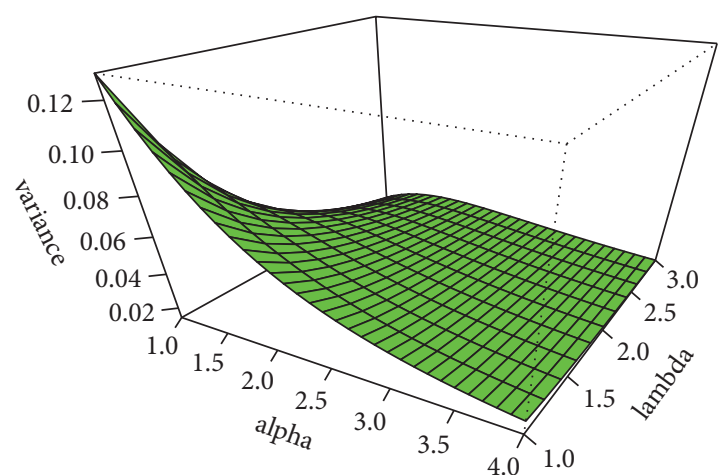

variance

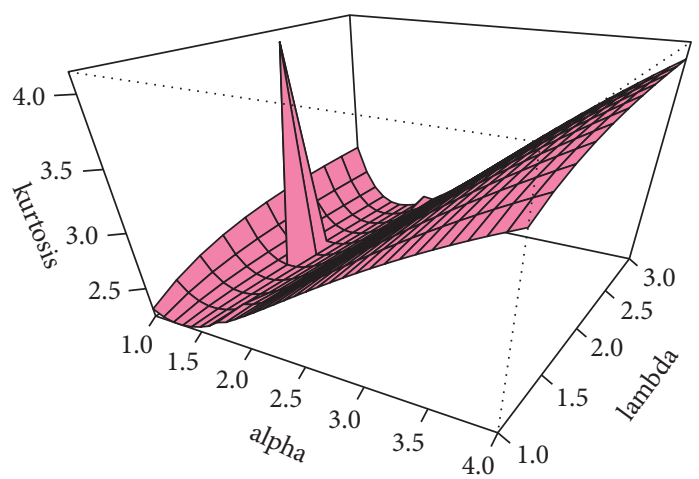

kurtosis

Figure 3: 3D plots of $M(Z), \operatorname{Var}(Z), \operatorname{CS}(Z)$, and $\mathrm{CK}(Z)$ of the OIPWU distribution for $\beta=\theta=2$.

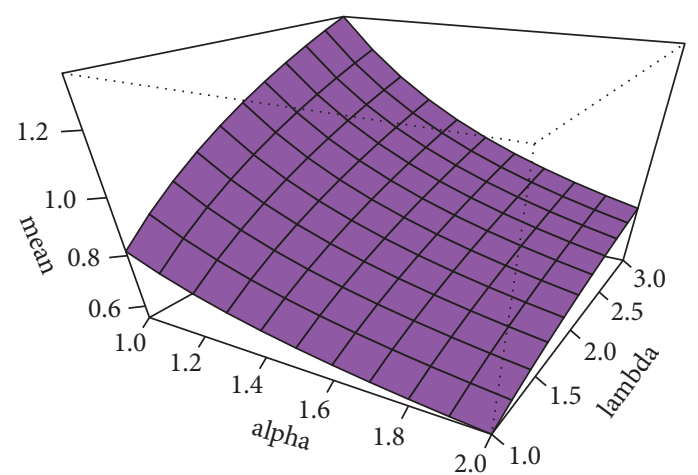

mean

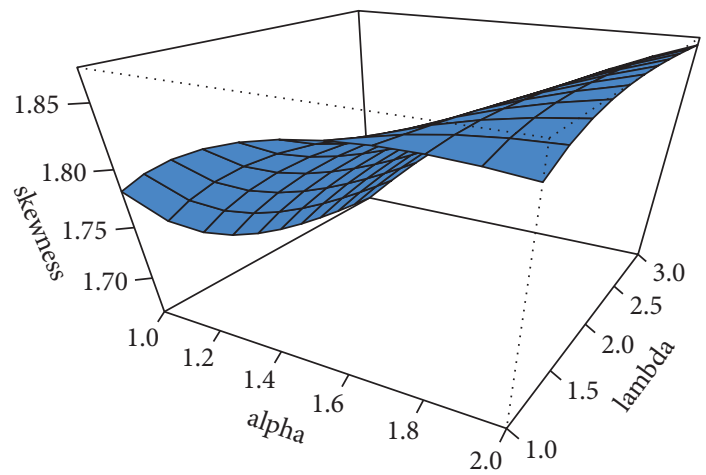

skewness

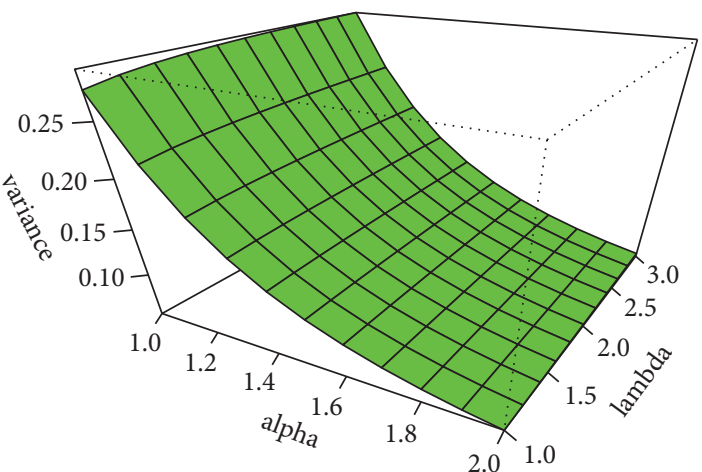

variance

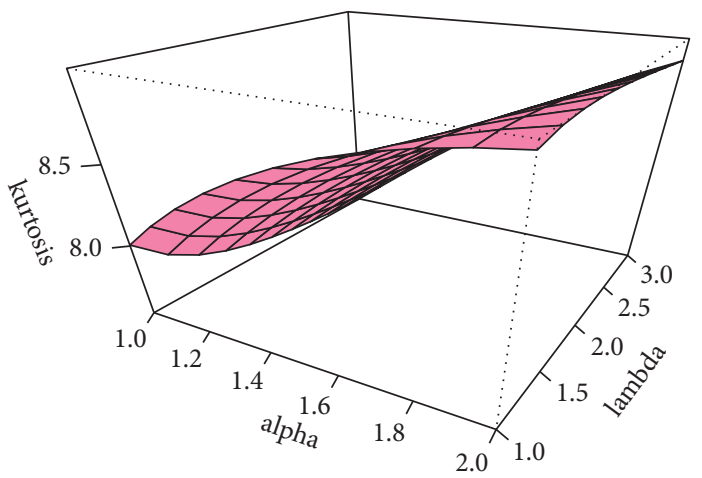

kurtosis

Figure 4: 3D plots of $M(Z), \operatorname{Var}(Z), \operatorname{CS}(Z)$, and $\operatorname{CK}(Z)$ of the OIPWEx distribution for $\beta=\theta=2$. 


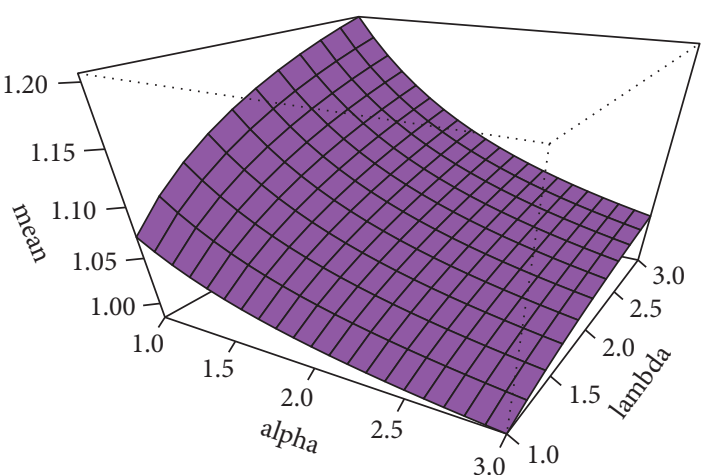

mean

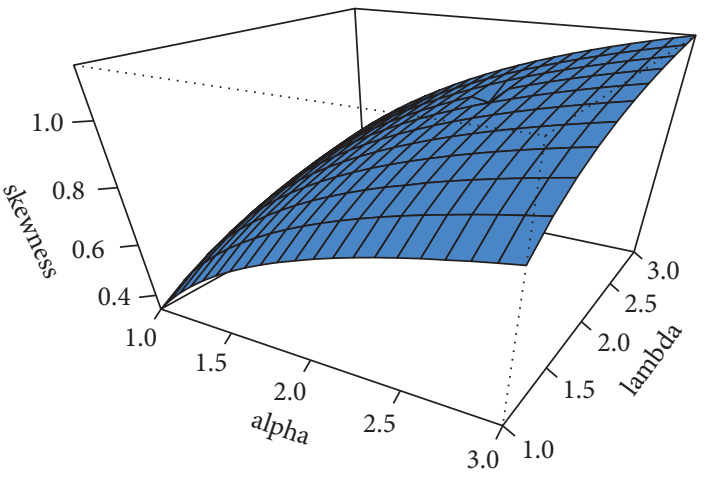

skewness

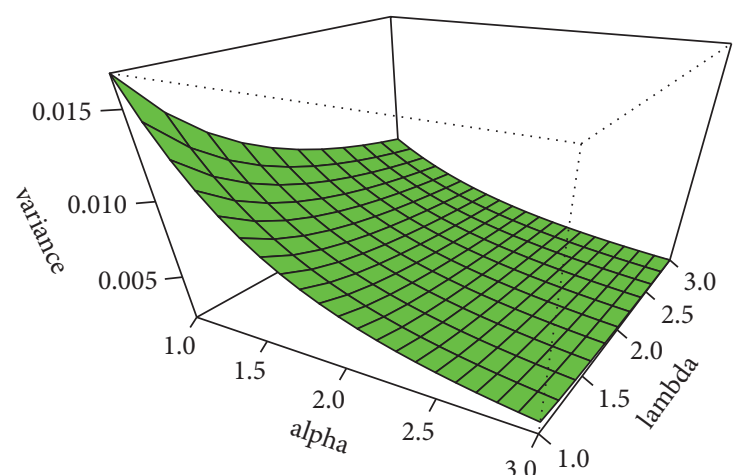

variance

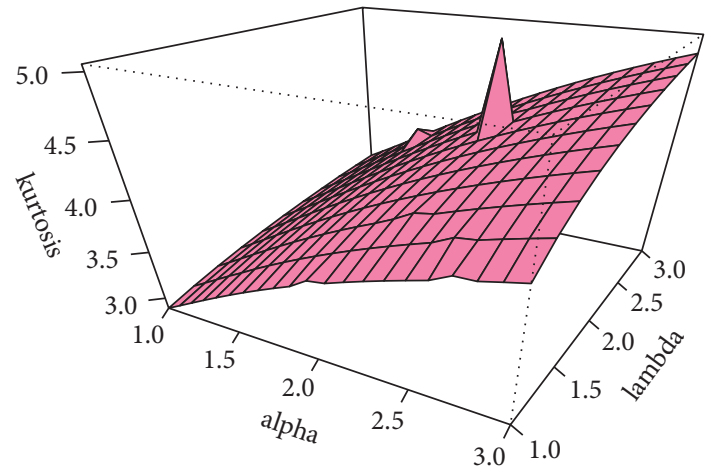

kurtosis

Figure 5: 3D plots of $M(Z), \operatorname{Var}(Z), \operatorname{CS}(Z)$, and $\mathrm{CK}(Z)$ of the OIPWW distribution for $\beta=5$ and $\theta=2$.
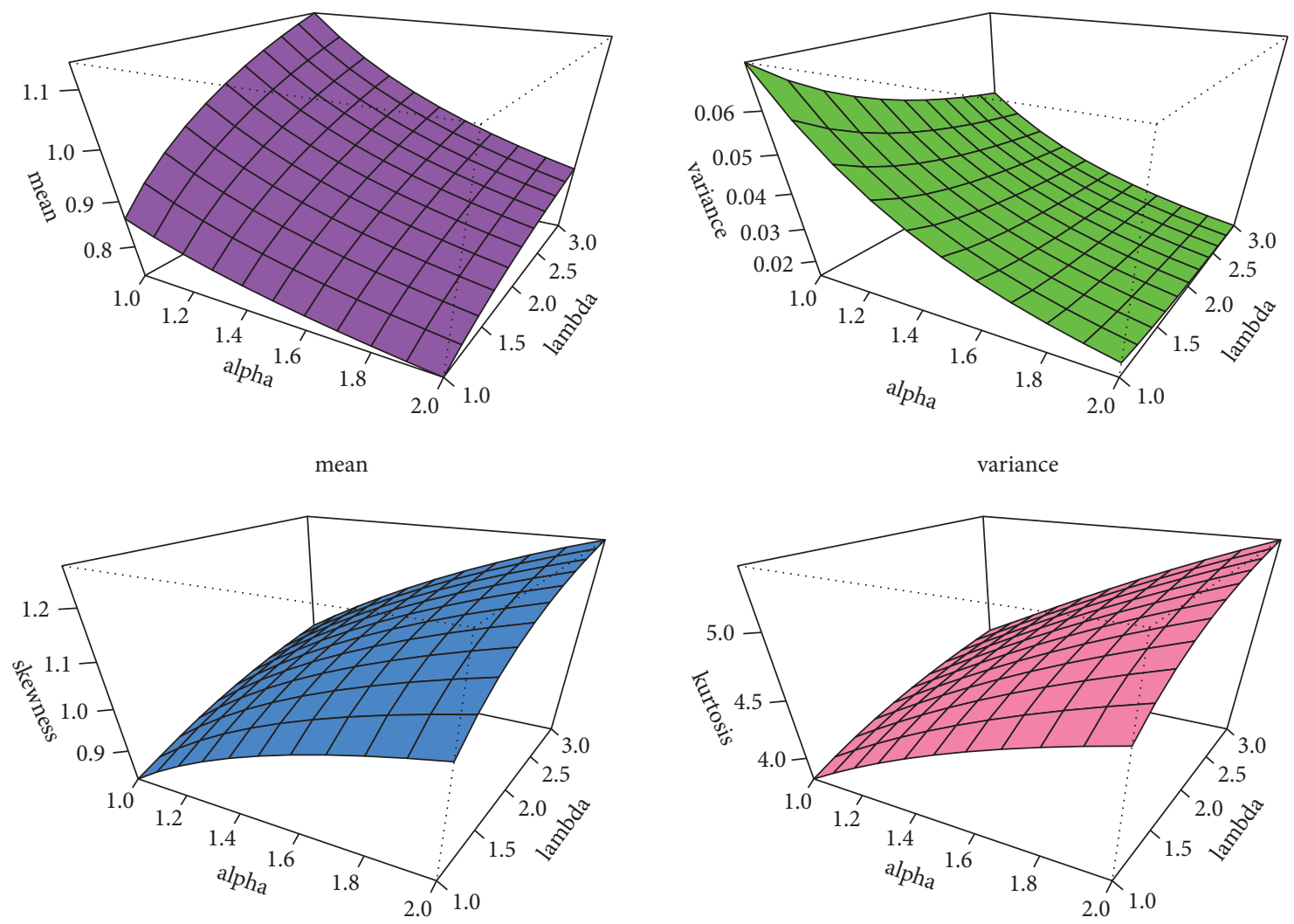

skewness

kurtosis

Figure 6: 3D plots of $M(Z), \operatorname{Var}(Z), \mathrm{CS}(Z)$, and $\mathrm{CK}(Z)$ of the OIPWR distribution for $\beta=\theta=2$. 


$$
I_{R}(\rho)=(1-\rho)^{-1} \log \left(\int_{-\infty}^{\infty}(f(z))^{\rho} \mathrm{d} z\right), \quad \rho \neq 1, \rho>0 . \quad f^{\rho}(z)=(\exp (1) \alpha \theta \lambda)^{\rho} \sum_{m=0}^{\infty} c_{m} g(z)^{\rho} G(z)^{m}
$$

where

Using (4), applying the same method of the useful expansion (14) and after some algebra, we get

$$
c_{m}=\sum_{\hbar, w, \kappa=0}^{\infty} \frac{\theta^{w+1}(-1)^{\hbar+w+m} \Gamma(\rho \alpha(\hbar+1)+w+\rho) \Gamma(\alpha(\rho+w)+\kappa+\rho)}{\hbar ! w ! \kappa ! \Gamma(\rho \alpha(\hbar+1)+\rho) \Gamma(\alpha(\rho+w)+\rho)}\left(\begin{array}{c}
\alpha(\rho+w)+\kappa-\rho \\
m
\end{array}\right) .
$$

Thus, Ré entropy of OIPGW-G family is given by

$$
\left.I_{R}(\rho)=\frac{\rho}{1-\rho} \log (\exp (1) \alpha \theta \lambda)+\frac{1}{1-\rho} \log \sum_{m=0}^{\infty} c_{m} \mathbf{I}_{-\infty}^{\infty}(z)\right],
$$

where $\mathbf{I}_{-\infty}^{\infty}(z)=\int_{-\infty}^{\infty} g(z)^{\rho} G(z)^{m} \mathrm{~d} z$.
The Tsallis entropy (TE) measure (see [19]) is defined by

$$
T_{R}(\rho)=\frac{1}{\rho-1}\left[1-\int_{-\infty}^{\infty}(f(z))^{\rho} \mathrm{d} z\right], \quad \rho \neq 1, \rho>0 .
$$

The Havrda and Charvat entropy $(\mathrm{HaChE})$ measure (see [20]) is defined by

$$
\operatorname{HaCh}_{R}(\rho)=\frac{1}{2^{1-\rho}-1}\left[\left(\int_{-\infty}^{\infty}(f(z))^{\rho} \mathrm{d} z\right)^{(1 / \rho)}-1\right], \quad \rho \neq 1, \rho>0
$$

The Arimoto entropy (ArE) measure (see [21]) of OIPGW-G is defined by

$$
\operatorname{Ar}_{R}(\rho)=\frac{\rho}{1-\rho}\left[\left(\int_{-\infty}^{\infty}(f(z))^{\rho} \mathrm{d} z\right)^{(1 / \rho)}-1\right], \quad \rho \neq 1, \rho>0 .
$$

Some of the numerical values of RéE, QE, HaChE, and ArE for a few chosen parameter values for the OIPGWEx model are given in Tables 5-8.

From Tables 3-8, we can note that the values of entropies can be negative or positive.

\section{Statistical Inference}

The parameters of the OIPGW-G are estimated using various methods including ML, LS, and WLS methods of estimation.

6.1. Maximum Likelihood Estimation. Let $z_{1}, \ldots, z_{n}$ be a $n$-sized random sample from the OIPGW-G with parameters $\alpha, \theta, \lambda$, and $\underline{\xi}$. Let $\Omega=(\alpha, \theta, \lambda, \underline{\xi})^{T}$ be $p \times 1$ vector of parameters. As an example, the log-likelihood function is defined as follows:

$$
\begin{aligned}
L_{n}= & n \log (\theta)+n \log (\alpha)+n \log (\lambda)+\sum_{\hbar=1}^{n} \log g_{\underline{\xi}}\left(z_{\hbar}\right)+(\alpha-1) \sum_{\hbar=1}^{n} \log \bar{G}_{\underline{\xi}}\left(z_{\hbar}\right) \\
& -(\alpha+1) \sum_{\hbar=1}^{n} \log \left(G_{\underline{\xi}}\left(z_{\hbar}\right)\right)+(\lambda-1) \sum_{\hbar=1}^{n} \log \left(1+\theta t_{\hbar}^{\alpha}\right)+\sum_{\hbar=1}^{n}\left\{1-\left[1+\theta t_{\hbar}^{\alpha}\right]^{\lambda}\right\},
\end{aligned}
$$

where $t_{\hbar}=\left(\bar{G}_{\xi}\left(z_{\hbar}\right) / G_{\xi}\left(z_{\hbar}\right)\right)$. The components of score vector $U(\Omega)={ }^{-}\left(\partial L_{n} / \partial \Omega\right)=\left(\left(\partial L_{n} / \partial \alpha\right),\left(\partial L_{n} / \partial \theta\right),\left(\partial L_{n} / \partial \lambda\right)\right.$, $\left.\left(\partial L_{n} / \partial \xi\right)\right)^{T}$ are given by 
TABle 5: Numerical values of RéE, QE, HaChE, and ArE at $\theta=\beta=\alpha=\rho=0.5$ for the OIPGWE model.

\begin{tabular}{lcccc}
\hline$\lambda$ & RéE & QE & HaChE & \\
\hline 0.2 & 1.027 & 1.342 & 1.157 & 1.792 \\
0.5 & 1.868 & 3.089 & 2.682 & 5.474 \\
0.8 & 2.235 & 4.116 & 3.873 & 8.351 \\
1.2 & 2.499 & 4.977 & 5.041 & 11.171 \\
1.5 & 2.619 & 5.41 & 5.686 & 12.728 \\
2 & 2.747 & 5.898 & 6.46 & 14.595 \\
\hline
\end{tabular}

TABle 6: Numerical values of RéE, QE, HaChE, and ArE at $\theta=\beta=\alpha=0.5$ and $\rho=1.5$ for the OIPGWEx model.

\begin{tabular}{lcccc}
\hline$\lambda$ & RéE & QE & HaChE & ArE \\
\hline 0.2 & -7.415 & -79.512 & -3.469 & -32.529 \\
0.5 & -0.83 & -1.028 & -0.386 & -0.956 \\
0.8 & 0.633 & 0.542 & -0.237 & 0.57 \\
1.2 & 1.475 & 1.043 & -0.179 & 1.165 \\
1.5 & 1.808 & 1.19 & -0.16 & 1.358 \\
2 & 2.118 & 1.306 & -0.145 & 1.519 \\
\hline
\end{tabular}

TABle 7: Numerical values of RéE, QE, HaChE, and ArE at $\theta=\beta=\lambda=\rho=0.5$ for the OIPGWEx model.

\begin{tabular}{lcccc}
\hline$\alpha$ & RéE & QE & HaChE & \\
\hline 0.3 & 2.145 & 3.846 & 3.539 & 7.544 \\
0.8 & 1.645 & 2.552 & 2.146 & 4.181 \\
1.2 & 1.436 & 2.101 & 1.742 & 3.205 \\
1.5 & 1.304 & 1.838 & 1.526 & 2.683 \\
2.0 & 1.111 & 1.486 & 1.258 & 2.037 \\
\hline
\end{tabular}

TAble 8: Numerical values of RéE, QE, HaChE, and ArE at $\theta=\beta=\lambda=0.5$ and $\rho=1.5$ for the OIPGWEx model.

\begin{tabular}{lcccc}
\hline$\alpha$ & RéE & QE & HaChE & \\
\hline 0.3 & -3.933 & -12.29 & -1.087 & -8.129 \\
0.8 & 0.307 & 0.285 & -0.264 & 0.292 \\
1.2 & 0.612 & 0.527 & -0.239 & 0.553 \\
1.5 & 0.628 & 0.539 & -0.238 & 0.566 \\
2 & 0.552 & 0.482 & -0.244 & 0.504 \\
\hline
\end{tabular}

$$
\begin{aligned}
U_{\alpha}= & \frac{\partial L_{n}}{\partial \alpha}=\frac{n}{\alpha}+\sum_{\hbar=1}^{n} \log \bar{G}_{\underline{\xi}}\left(z_{\hbar}\right)-\sum_{\hbar=1}^{n} \log \left[G_{\underline{\xi}}\left(z_{\hbar}\right)\right] \\
& +(\lambda-1) \sum_{\hbar=1}^{n} \frac{\theta t_{\hbar}^{\alpha} \log t_{\hbar}}{\left(1+\theta t_{\hbar}^{\alpha}\right)}-\lambda \sum_{\hbar=1}^{n} \theta t_{\hbar}^{\alpha} \log \left(t_{\hbar}\right)\left(1+\theta t_{\hbar}^{\alpha}\right)^{\lambda-1}, \\
U_{\theta}= & \frac{\partial L_{n}}{\partial \theta}=\frac{n}{\theta}+(\lambda-1) \sum_{\hbar=1}^{n} \frac{t_{\hbar}^{\alpha}}{1+\theta t_{\hbar}^{\alpha}}-\lambda \sum_{\hbar=1}^{n} t_{\hbar}^{\alpha}\left(1+\theta t_{\hbar}^{\alpha}\right)^{\lambda-1}, \\
U_{\lambda}= & \frac{\partial L_{n}}{\partial \lambda}=\frac{n}{\lambda}+\sum_{\hbar=1}^{n} \log \left(1+\theta t_{\hbar}^{\alpha}\right)-\sum_{\hbar=1}^{n}\left(1+\theta t_{\hbar}^{\alpha}\right)^{\lambda} \log \left(1+\theta t_{\hbar}^{\alpha}\right), \\
U_{\underline{\xi}_{\kappa}}= & \frac{\partial L_{n}}{\partial \underline{\xi}_{\kappa}}=\sum_{\hbar=1}^{n} \frac{g_{\underline{\xi}}^{\prime}\left(z_{\hbar}\right)}{G_{\underline{\xi}}\left(z_{\hbar}\right)}+(\alpha-1) \sum_{\hbar=1}^{n} \frac{G_{\underline{\xi}}^{\prime}\left(z_{\hbar}\right)}{\bar{G}_{\underline{\xi}}\left(z_{\hbar}\right)}-(\alpha+1) \sum_{\hbar=1}^{n} \frac{G_{\underline{\xi}}^{\prime}\left(z_{\hbar}\right)}{G_{\underline{\xi}}\left(z_{\hbar}\right)} \\
& +(\lambda-1) \sum_{\hbar=1}^{n} \frac{\theta \alpha t_{\hbar}^{\alpha-1}}{1+\theta t_{\hbar}^{\alpha}} \partial t_{\hbar} \partial \underline{\xi}_{\kappa}-\lambda \sum_{\hbar=1}^{n} \theta \alpha t_{\hbar}^{\alpha-1}\left(1+\theta t_{\hbar}^{\alpha}\right)^{\lambda-1} \partial t_{\hbar} \partial \underline{\xi}_{\kappa},
\end{aligned}
$$

where $g_{\xi}^{\prime}\left(z_{\hbar}\right)=\left(\partial G_{\xi}\left(z_{\hbar}\right) / \partial \underline{\xi}_{\kappa}\right), G_{\xi}^{\prime}\left(z_{\hbar}\right)=\left(\partial G_{\xi}\left(z_{\hbar}\right) / \partial \underline{\xi}_{\kappa}\right)$, and $\bar{G}_{\xi}^{\prime}\left(z_{\hbar}^{-}\right)=\left(\partial \bar{G}_{\xi}\left(z_{\hbar}^{-}\right) / \partial \underline{\xi}_{\kappa}\right)$ and $\underline{\xi}_{\kappa}^{-}$is the $\kappa_{\text {th }}$ element of the vector of parameters $\xi$. The maximum likelihood estimation (MLE) of parameters $\alpha, \theta, \lambda$, and $\xi$ is obtained by setting $U_{\alpha}=U_{\theta}=U_{\lambda}=U_{\xi}=0$ and solving these equations simultaneously to get the $\operatorname{MLE}(\widehat{\Omega})=\left(\widehat{\alpha}, \widehat{\theta}, \widehat{\lambda}, \widehat{\xi}^{T}\right)^{T}$ of $\Omega$.

6.2. Ordinary and Weighted Least Square Estimators. Suppose $z_{1}, \ldots, z_{n}$ is a random sample from OIPGW-G with corresponding ordered sample of $z_{(1)}, \ldots, z_{(n)}$. The mean and variance of OIPGW-G are independent of unknown parameter and are as follows: $E\left(F\left(Z_{(i)}\right)\right)=(i /(n+1))$ and $\operatorname{var}\left(F\left(Z_{(i)}\right)=\left(i(n-i+1) /(n+1)^{2}(n+2)\right)\right.$, where $F\left(Z_{(i)}\right)$ is cdf of OIPGW-G with be the $i^{\text {th }}$ order statistic. Then, LS estimators are obtained by minimizing the SSE:

$$
\sum_{i=1}^{n}\left[F_{(i)}(z)-\frac{i}{n+1}\right]^{2}
$$

with respect to $\Omega$.

The WLS estimators of $\Omega$ can be obtained by minimizing the following expression: 


$$
\sum_{i=1}^{n} \frac{(n+1)^{2}(n+2)}{i(n-i+1)}\left[F_{(i)}(z)-\frac{i}{n+1}\right]^{2}
$$

with respect to $\Omega$.

6.3. Simulation Outcomes. Here, we come up with a numerical study to compare the behavior of different estimates. We generate 1000 random samples of size $n=50,100$, and 200 from the OIPGWEx distribution from the following equation:

$$
Q(u)=\frac{-1}{\beta} \ln \left[1-\left(1+\left\{\frac{1}{\theta}\left[(1-\ln u)^{(1 / \lambda)}-1\right]\right\}^{(1 / \alpha)}\right)^{-1}\right]
$$

Three sets of the parameters are assigned. The MLE, LSE, and WLSE of $\alpha, \theta, \lambda$, and $\beta$ are determined by using MATHCAD (14). Then, the estimates of all methods and their mean square errors (MSEs) are documented in Tables 9-11.

\section{Copula under the OIPGWEx Model}

The fact that $C .(u, v)$ is a straightforward function of the $\mathrm{U}$ marginal CDFs, $F(u)$ and $G(v)$, is a property shared by all of the probability distributions in this section. These sorts of joint models are referred to as "Copulas (Co)." Copula techniques are widely used in insurance, econometrics, and finance. They are multivariate distributions with uniform marginals on the interval $I_{(0,1)}=(0,1)$. Using FGM Co, modified FGM Co, Clayton Co, and Rényi's entropy, we construct several new bivariate type OIPGWEx (BvOIPGWEx) models. Additionally, the MvOIPGWEx type of the multivariate $\mathrm{KBX}$ is discussed. These new models, on the other hand, may be the subject of future research efforts.

7.1. FGM Copula. For starters, let us look at the combined CDF of two RVs $\left(W_{1}, W_{2}\right)$ for Morgenstern family [22] which is characterized by the following $\left.C_{\lambda}(m, \omega)\right|_{\left(\lambda \in \mathbf{I}_{(-1,1)} \text { and } m, \omega \in(0,1)\right)}=(1+\lambda \bar{m} \omega) m \omega . \quad$ Sett $\bar{m}=1-m$ and $\bar{\omega}=1-\omega$. Then, $\bar{m}=\bar{F}_{\mathbf{V}_{1}}\left(w_{1}\right)$ and $\bar{\omega}=\bar{F}_{\underline{\mathbf{V}_{2}}}\left(w_{2}\right)$. Then, we have $C_{\lambda}(m, \omega)=C_{\lambda}\left(\frac{w_{1}}{w_{1}}, w_{2}\right)$.

7.2. Modified FGM Copula. This modified FGM Co (see [23-25]) is a good example of this.

$$
\left.C_{\Delta}(m, \omega)\right|_{\Delta(-1,1)}=m \omega[1+\Delta \vartheta(m) \widetilde{\delta}(\omega)]=m \omega+\Delta \widetilde{\vartheta(m)} \widetilde{\sigma(\omega)},
$$

where $(\widetilde{\vartheta(m)} / m)=\vartheta(m)$ and $(\widetilde{\sigma(\omega)} / \omega)=\mho(\omega) . \vartheta(m)$ and $\delta(\omega)$ consist of two activities that are inseparable $\mathbf{I}_{(0,1)}$ where $\vartheta(0)=\sigma(0)=\sigma(1)=\vartheta(1)=0$. Let
TAble 9: MLEs, LSEs, WLSEs, and MSEs at Set $1: \alpha=\lambda=\theta=\beta=$ 0.5 for the OIPGWEx model.

\begin{tabular}{ccccccc}
\hline$n$ & MLEs & MSEs & LSEs & MSEs & WLSEs & MSEs \\
\hline \multirow{4}{*}{50} & 0.519 & 0.042 & 0.426 & 0.0114 & 0.491 & 0.0761 \\
& 0.629 & 0.071 & 0.706 & 0.0596 & 0.586 & 0.0195 \\
& 0.465 & 0.062 & 0.479 & 0.0418 & 0.509 & 0.0287 \\
& 0.622 & 0.128 & 0.895 & 0.4903 & 0.632 & 0.0651 \\
\hline \multirow{4}{*}{100} & 0.493 & 0.017 & 0.434 & 0.0092 & 0.508 & 0.0029 \\
& 0.602 & 0.049 & 0.685 & 0.0515 & 0.576 & 0.0108 \\
& 0.47 & 0.03 & 0.481 & 0.0226 & 0.508 & 0.0165 \\
& 0.56 & 0.062 & 0.715 & 0.1323 & 0.621 & 0.0377 \\
\hline \multirow{4}{*}{200} & 0.5 & 0.0086 & 0.451 & 0.0088 & 0.494 & 0.0028 \\
& 0.561 & 0.025 & 0.613 & 0.0445 & 0.566 & 0.0054 \\
& 0.461 & 0.023 & 0.486 & 0.0147 & 0.504 & 0.0085 \\
& 0.525 & 0.028 & 0.671 & 0.1244 & 0.571 & 0.0317 \\
\hline
\end{tabular}

TABle 10: MLEs, LSEs, WLSEs, and MSEs at Set 2: $\alpha=\theta=\beta=0.5$ and $\lambda=1.2$ for the OIPGWEx model.

\begin{tabular}{ccccccc}
\hline$n$ & MLEs & MSEs & LSEs & MSEs & WLSEs & MSEs \\
\hline \multirow{4}{*}{50} & 0.519 & 0.016 & 0.502 & 0.0075 & 0.555 & 0.0122 \\
& 1.378 & 0.183 & 1.098 & 0.0178 & 1.138 & 0.0457 \\
& 0.482 & 0.063 & 0.574 & 0.0261 & 0.435 & 0.032 \\
& 0.559 & 0.066 & 0.539 & 0.0433 & 0.384 & 0.0358 \\
\hline \multirow{4}{*}{100} & 0.522 & 0.01 & 0.508 & 0.00518 & 0.549 & 0.0117 \\
& 1.288 & 0.149 & 1.109 & 0.0132 & 1.172 & 0.0269 \\
& 0.495 & 0.037 & 0.561 & 0.0179 & 0.436 & 0.0243 \\
& 0.51 & 0.026 & 0.527 & 0.026 & 0.391 & 0.0344 \\
\hline \multirow{4}{*}{200} & 0.521 & 0.0089 & 0.511 & 0.00286 & 0.535 & 0.009 \\
& 1.234 & 0.139 & 1.106 & 0.0116 & 1.236 & 0.0255 \\
& 0.534 & 0.037 & 0.553 & 0.0105 & 0.463 & 0.0193 \\
& 0.513 & 0.018 & 0.507 & 0.0128 & 0.396 & 0.0258 \\
\hline
\end{tabular}

$$
\begin{aligned}
& \alpha=\inf \left\{\frac{\partial}{\partial m} \widetilde{\vartheta(m)}: \mathscr{W}_{1}(m)\right\}<0, \\
& \underline{\xi}=\inf \left\{\frac{\partial}{\partial \omega} \widetilde{\mho(\omega)}: \mathscr{W}_{2}(\omega)\right\}>0, \\
& \beta=\sup \left\{\frac{\partial}{\partial m} \widetilde{\vartheta(m)}: \mathscr{W}_{1}(m)\right\}<0, \\
& \eta=\sup \left\{\frac{\partial}{\partial \omega} \widetilde{\mho(\omega)}: \mathscr{W}_{2}(\omega)\right\}>0,
\end{aligned}
$$

and $\min (\alpha \beta, \underline{\xi} \eta) \geq 1$ where

$$
\begin{aligned}
\frac{\partial}{\partial m} \widetilde{\vartheta(m)} & =\vartheta(m)+m \frac{\partial}{\partial m} \vartheta(m), \\
\mathscr{W}_{1}(m) & =\left\{m: m \in \mathbf{I}_{(0,1)} \mid \frac{\partial}{\partial m} \widetilde{\vartheta(m)} \text { exists }\right\}, \\
\mathscr{W}_{2}(\omega) & =\left\{\omega: \omega \in \mathbf{I}_{(0,1)} \mid \frac{\partial}{\partial \omega} \widetilde{\widetilde{\sigma(\omega)}} \text { exists }\right\} .
\end{aligned}
$$


TAble 11: MLEs, LSEs, WLSEs, and MSEs at Set 3: $\theta=\beta=0.5$ and $\alpha=\lambda=1.2$ for the OIPGWEx model.

\begin{tabular}{|c|c|c|c|c|c|c|}
\hline$n$ & MLEs & MSEs & LSEs & MSEs & WLSEs & MSEs \\
\hline \multirow{4}{*}{50} & 1.378 & 0.107 & 1.374 & 0.0811 & 1.345 & 0.0511 \\
\hline & 1.233 & 0.249 & 1.315 & 0.2051 & 1.128 & 0.0495 \\
\hline & 0.385 & 0.083 & 0.396 & 0.0521 & 0.376 & 0.0415 \\
\hline & 0.416 & 0.056 & 0.386 & 0.0276 & 0.358 & 0.0219 \\
\hline \multirow{4}{*}{100} & 1.388 & 0.075 & 1.36 & 0.0682 & 1.318 & 0.0239 \\
\hline & 1.185 & 0.202 & 1.222 & 0.1965 & 1.113 & 0.0242 \\
\hline & 0.386 & 0.054 & 0.401 & 0.0516 & 0.395 & 0.0381 \\
\hline & 0.422 & 0.026 & 0.391 & 0.0265 & 0.378 & 0.0155 \\
\hline \multirow{4}{*}{200} & 1.347 & 0.061 & 1.342 & 0.0602 & 1.277 & 0.0113 \\
\hline & 1.188 & 0.141 & 1.199 & 0.1851 & 1.2 & 0.0157 \\
\hline & 0.387 & 0.044 & 0.411 & 0.048 & 0.448 & 0.0278 \\
\hline & 0.459 & 0.028 & 0.466 & 0.0251 & 0.476 & 0.0107 \\
\hline
\end{tabular}

7.2.1. Bivariate OIPGWEx-FGM (Type-I) Model. The bivariate OIPGWEx-FGM (Type-I) model may be constructed directly from $C_{\Delta}(m, \omega)=m \omega+\Delta \widetilde{\vartheta(m)} \widetilde{\sigma(\omega)}$ where $\widetilde{\vartheta(m)}=$ $m \bar{F}_{\underline{\mathbf{V}_{1}}}(m)$ and $\tilde{\sigma(\omega)}=\omega \bar{F}_{\underline{\mathbf{V}_{2}}}(\omega)$.

\subsubsection{Bivariate OIPGWEx-FGM (Type-II) Model.} Suppose that $\vartheta(m)$ and $\delta(\omega)$ meet all of the criteria mentioned previously, and

$$
\begin{aligned}
& \left.\vartheta^{*}(m)\right|_{\left(\Delta_{1}>0\right)}=m^{\Delta_{1}}(1-m)^{1-\Delta_{1}} \\
& \left.\mho^{*}(\omega)\right|_{\left(\Delta_{2}>0\right)}=\omega^{\Delta_{2}}(1-\omega)^{1-\Delta_{2}} .
\end{aligned}
$$

The corresponding bivariate Co (henceforth, bivariate OIPGWEx-FGM (Type-II) Co) can be deduced:

$$
\left.C_{\Delta, \Delta_{1}, \Delta_{2}}(m, \omega)\right|_{\Delta \in \mathbf{I}_{(-1,1)}}=m \omega\left[1+\Delta \vartheta^{*}(m) \mho^{*}(\omega)\right] .
$$

7.2.3. Bivariate OIPGWEx-FGM (Type-III) Model. For both, $\vartheta(m)$ and $\delta(\omega)$ have a look at the form below. You may calculate the CDF of the bivariate CDF OIPGWEx-FGM (Type-III) model using $\vartheta(m), \mho(\omega), \vartheta(m)=m[\log (1+\bar{m})]$, and $\sigma^{\cdot}(\omega)=\omega[\log (1+\bar{\omega})]$ which can be computed as

$$
C_{\Delta}(m, \omega)=m \omega\left(1+\Delta \vartheta(m) \mho^{\prime}(\omega)\right) .
$$

7.2.4. Bivariate OIPGWEx-FGM (Type-IV) Model. You may calculate the CDF of the bivariate OIPGWEx-FGM (TypeIV) model using $C_{\Delta}(m, \omega)=\omega F^{-1}(m)+m F^{-1}(\omega)-\left[F^{-1}(m) F^{-1}(\omega)\right]$ where $F^{-1}(m)$ and $F^{-1}(\omega)$ can be computed from (6).

7.3. The Bivariate OIPGWEx via Rényi's Entropy. Following [26], the joint CDF of the bivariate OIPGWEx via RéE can be written as $C(m, \omega)=w_{2} m+w_{1} \omega-w_{1} w_{2}$. Let $m=1-\bar{F}_{\mathbf{V}_{1}}\left(w_{1}\right) \in \mathbf{I}_{(0,1)}$ and $\omega=1-\bar{F}_{\mathbf{V}_{2}}\left(w_{2}\right) \in \mathbf{I}_{(0,1)}$; the associated bivariate OIPGWEx will be $C\left(w_{1}, w_{2}\right)=$ $C(m, \omega)$.

7.4. The Bivariate OIPGWEx Extension via Clayton Copula. Bivariate extension through Clayton Copula is a weighted variant of the Clayton Co, which has the form
$\left.C(m, \omega)\right|_{[\eta \geq 0]}=\left[m^{-\eta}+\omega^{-\eta}-1\right]^{-(1 / \eta)} \quad$ where $m=1-\bar{F}_{\mathbf{V}_{1}}\left(w_{1}\right) \in \mathbf{I}_{(0,1)} \quad$ and $\quad \omega=1-\bar{F}_{\mathbf{V}_{2}}\left(w_{2}\right) \in \mathbf{I}_{(0,1)}$. Similarly, the multivariate OIPGWE extension can be obtained from $C\left(w_{1}, w_{2}, \ldots, w_{D}\right)=\left(\sum_{i=1}^{D} m_{i}^{-\eta}+1-D\right)^{-(1 / \eta)}$ where $m_{i}=1-\bar{F}_{\underline{\mathbf{V}_{i}}}\left(w_{i}\right) \in \mathbf{I}_{(0,1)}$.

\section{Real Data Applications}

Comparison of the OIPGWEx distribution with various competing models will be made particularly exponential (Ex), odd Lindley Ex (OLEx), Marshall-Olkin (MO) Ex (MOEx), moment exponential (MEx), the logarithmic Burr-Hatke Ex (LBHEx), generalized MO Ex (GMOEx), beta Ex (BEx), MO Kumaraswamy Ex (MOKwEx), Kumaraswamy exponential (KwEx), the Burr X Ex (BrXEx), and Kw MO Ex (KwMOEx). Some details related to these competitive models are available in [27]. For comparing models, we consider the Cramér-Von Mises $\left(C^{*}\right)$ and the Akaike information criterion (AIC) $\left(C_{1}\right)$, Bayesian IC $\left(C_{2}\right), \quad$ Anderson-Darling ((HTML translation failed)), Consistent AIC $\left(C_{3}\right)$, the Kolmogorov-Smirnov (K. S.), Hannan-Quinn IC $\left(C_{4}\right)$, and $p$ value (P. V.) statistics.

8.1. Modeling Failure (Relief) Times. The failure time data are the first data set. Recent analyses of these data were conducted by [27]. Table 12 lists the MLEs. Table 13 lists $C_{1}, C_{2}$, $C_{3}, C_{4}, A^{*}, C$, K. S., and P. V. The total time test (TTT) plot for the relief time data, as well as the related box plot, is shown in Figure 7. Based on Figure 7, the HRF of the relief times is "increasing HRF," and these data have only one EV observation. Figure 8 gives the E-PDF, E-CDF, E-HRF, and P-P plot for relief time data. Figure 9 gives Kaplan-Meier (KM) survival plot for relief time data.

Based on Table 13, we have come to the conclusion that the OIPGWEx model is much better than the Ex, OLEx, MOEx, MEx, LBHEx, GMOEx, BEx, MOKwEx, KwE, BXEx, and KwMOEx models with $C_{1}=38.74, C_{2}=42.7, C_{3}=41.41$, $C_{4}=39.52, \mathbb{A}^{*}=0.178, \quad C^{*}=0.0317, \quad K . S=0.0999$, and P. V. $=0.9884$, so the OIPGWEx model is an excellent alternative to these competitive distributions in modeling relief times data set. According to Figures 8 and 9, the 
TABle 12: MLEs values for the first data.

\begin{tabular}{|c|c|}
\hline Models & MLEs \\
\hline $\operatorname{Ex}_{(\beta)}$ & 0.526 \\
\hline $\operatorname{OLEx}_{(\beta)}$ & 0.6044 \\
\hline $\operatorname{MEx}_{(\beta)}$ & 0.950 \\
\hline $\operatorname{LBHEx}_{(\beta)}$ & 0.5263 \\
\hline $\operatorname{MOEx}_{(\alpha, \beta)}$ & $54.474,2.316$ \\
\hline $\operatorname{BrXEx}_{(a, \beta)}$ & $1.1635,0.3207$ \\
\hline $\operatorname{GMOEx}_{(\lambda, \alpha, \beta)}$ & $0.519,89.462,3.169$ \\
\hline $\operatorname{KwEx}_{(\alpha, \theta, \beta)}$ & $83.756,0.568,3.330$ \\
\hline $\operatorname{BEx}_{(\alpha, \theta, \beta)}$ & $81.633,0.542,3.514$ \\
\hline $\operatorname{MOKwEx}_{(\lambda, \alpha, \theta, \beta)}$ & $0.13,33.24,0.57,1.67$ \\
\hline $\operatorname{KwMOEx}_{(\lambda, \alpha, \theta, \beta)}$ & $8.87,34.83,0.29,4.89$ \\
\hline $\operatorname{OIPGWEx}_{(\lambda, \alpha, \theta, \beta)}$ & $1.971,2.36,0.51,0.47$ \\
\hline
\end{tabular}

Table 13: $C_{1}, C_{2}, C_{3}, C_{4}, A^{j}, C$, K. S., and P. V. for the first data.

\begin{tabular}{|c|c|c|c|c|c|}
\hline Models & $C_{1}, C_{2}, C_{3}, C_{4}$ & $\mathbb{A}^{\circ}$ & $C$ & K. S. & (P. V.) \\
\hline Ex & $67.70,68.70,67.89,68.90$ & 4.60 & 0.96 & 0.44 & $(<0.01)$ \\
\hline OLEx & $49.12,50.14,49.33,49.34$ & 1.3 & 0.22 & 0.85 & $(<0.001)$ \\
\hline MEx & $54.32,55.31,54.54,54.50$ & 2.76 & 0.53 & 0.32 & $(0.1)$ \\
\hline LBHEx & $67.70,68.70,67.89,67.90$ & 0.62 & 0.105 & 0.44 & $(<0.001)$ \\
\hline MOEx & $43.51,45.51,44.22,43.90$ & 0.8 & 0.14 & 0.18 & $(0.55)$ \\
\hline GMOEx & $42.75,45.74,44.25,43.34$ & 0.51 & 0.08 & 0.15 & $(0.78)$ \\
\hline $\mathrm{KwEx}$ & $41.78,44.75,43.28,42.32$ & 0.45 & 0.07 & 0.14 & $(0.86)$ \\
\hline BEx & $43.48,46.45,44.98,44.02$ & 0.70 & 0.12 & 0.16 & $(0.80)$ \\
\hline MOKEx & $41.58,45.54,44.25,42.30$ & 0.60 & 0.11 & 0.14 & $(0.87)$ \\
\hline KMOEx & $42.82,46.84,45.55,43.60$ & 1.08 & 0.19 & 0.15 & $(0.86)$ \\
\hline BrXEx & $48.13,50.15,48.83,48.52$ & 1.39 & 0.24 & 0.248 & $(0.171)$ \\
\hline OIPGWEX & $38.74,42.7,41.41,39.52$ & 0.178 & 0.0317 & 0.0999 & $(0.9884)$ \\
\hline
\end{tabular}

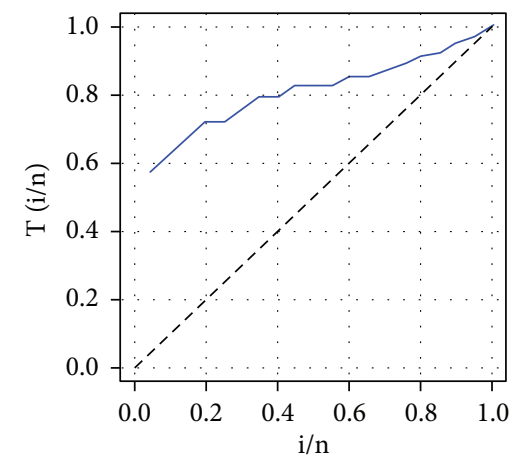

(a)

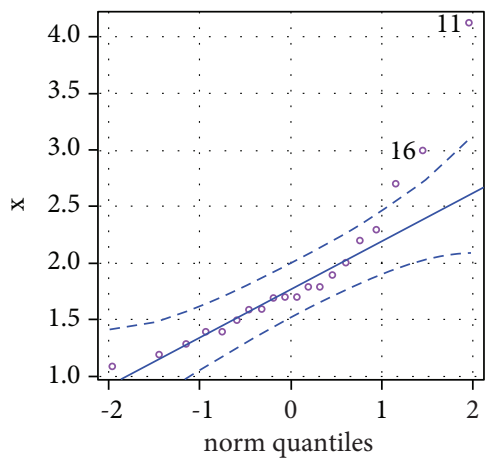

(b)

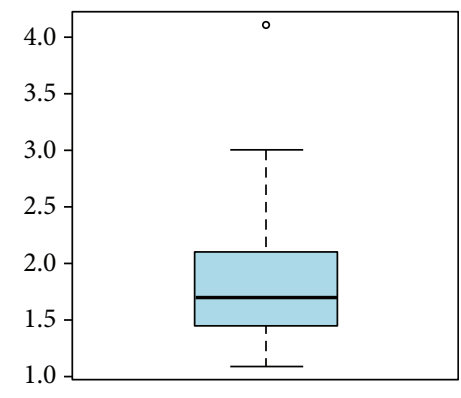

(c)

Figure 7: The TTT plot (a), Q-Q plot (b), and box plot (c) for the first data.

OIPGWEx distribution provides adequate fits to the empirical functions.

8.2. Modeling Survival times. The survival data are the second data set. Recent analysis of these data was conducted by [29]. Table 14 lists the MLEs. Table 15 lists the $C_{1}, C_{2}, C_{3}$, $C_{4}, A^{*}, C$, K. S., and P. V. Figure 10 gives the TTT plot along with the corresponding box plot for the survival time data.
Based on Figure 10, the HRF of the survival times is "increasing HRF," and these data have only four EV observations. Figure 11 gives the estimated PDF (E-PDF), E-CDF, E-HRF, and P-P plot survival time data. Figure 12 gives the Kaplan-Meier survival plot survival times data. Based on Table 15, we have come to the conclusion that the OIPGWEx model is far superior to the Ex, OLEx, MOEx, MEx, LBHEx, GMOEx, BEx, MOKwEx, KwEx, BXEx, and KwMOEx models with $C_{1}=206.88, C_{2}=215.98, C_{3}=207.47$, 


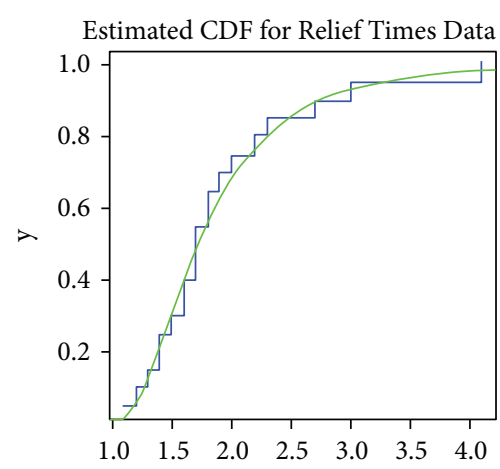

— The OIPGWE Distribution

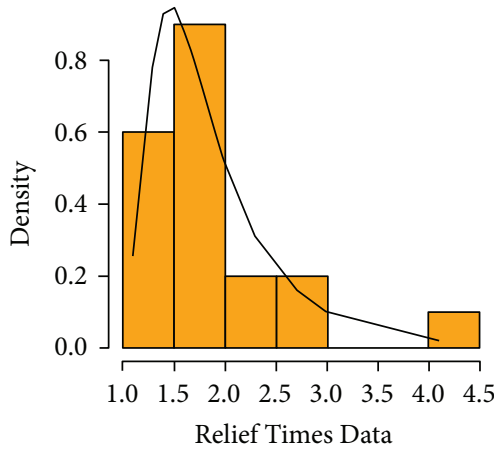

- The OIPGWE Distribution

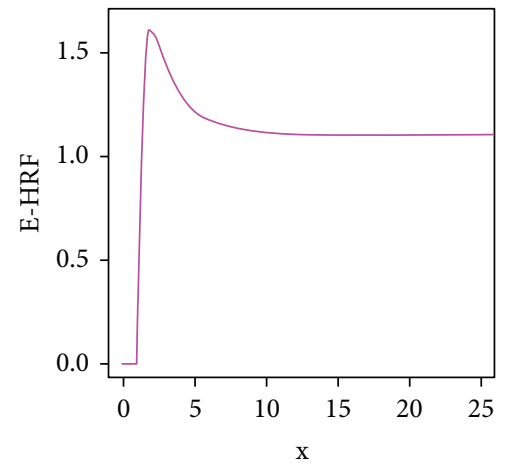

Figure 8: E-CDF, E-PDF, and E-HRF for the first data.
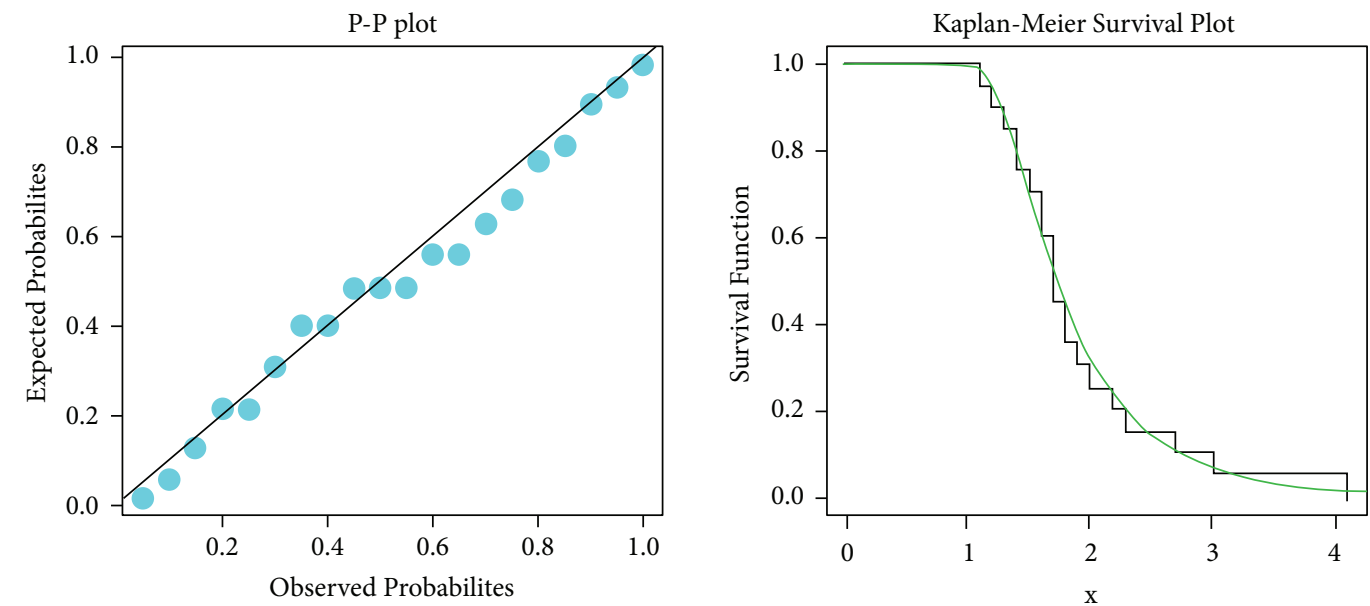

FIgURE 9: P-P plot and KM survival plot the first data.

TABLE 14: MLEs values for the second data.

\begin{tabular}{|c|c|}
\hline Models & MLEs \\
\hline $\mathrm{Ex}_{(\beta)}$ & 0.540 \\
\hline $\operatorname{OLEx}_{(\beta)}$ & 0.3815 \\
\hline $\operatorname{MEx}_{(\beta)}$ & 0.925 \\
\hline $\operatorname{LBHEx}_{(\beta)}$ & 0.54 \\
\hline $\operatorname{MOEx}_{(\alpha, \beta)}$ & $8.78,1.38$ \\
\hline $\operatorname{BrXEx}_{(a, \beta)}$ & $0.48,0.21$ \\
\hline $\operatorname{GMOEx}_{(\lambda, \alpha, \beta)}$ & $0.18,47.64,4.47$ \\
\hline $\operatorname{KwEx}_{(a, \theta, \beta)}$ & $3.304,1.100,1.037$ \\
\hline $\operatorname{BEx}_{(\alpha, \theta, \beta)}$ & $0.807,3.461,1.331$ \\
\hline $\operatorname{MOKwEx}_{(\alpha, \beta, \lambda, \beta)}$ & $0.01,2.72,1.99,0.01$ \\
\hline $\operatorname{KwMOEx}_{(\alpha, \beta, \lambda, \beta)}$ & $0.37,3.48,3.31,0.29$ \\
\hline $\operatorname{OIPGWEx}_{(\lambda, \alpha, \theta, \beta)}$ & $1.146,0.26,4.07,4.5$ \\
\hline
\end{tabular}

$C_{4}=210.50, \quad \mathbb{A}^{*}=0.63, \quad C=0.105, \quad$ K. S. $=0.088, \quad$ and P. V. $=0.639$, so the OIPGWEx model is an excellent alternative to these competitive distributions in modeling relief times data set. According to Figures 11 and 12, the OIPGWEx distribution provides adequate fits to the empirical functions. 
TAble 15: $C_{1}, C_{2}, C_{3}, C_{4}, A^{*}, C, K$. S., and P. V. for second data.

\begin{tabular}{|c|c|c|c|c|c|}
\hline Models & $C_{1}, C_{2}, C_{3}, C_{4}$ & $\mathbb{A}^{\circ}$ & $C$ & K. S. & (P. V.) \\
\hline $\mathrm{Ex}$ & $234.60,236.90,234.68,235.55$ & 6.53 & 1.25 & 0.3 & $(0.06)$ \\
\hline OLEx & $229.13,231.43,229.21,230.11$ & 1.94 & 0.33 & 0.5 & $(<0.001)$ \\
\hline $\mathrm{ME}$ & $210.40,212.68,210.45,211.30$ & 1.52 & 0.25 & 0.15 & $(0.13)$ \\
\hline LBHEx & $234.63,236.92,234.71,235.51$ & 0.71 & 0.115 & 0.28 & $(<0.001)$ \\
\hline MOEx & $210.37,214.93,210.52,212.17$ & 1.18 & 0.17 & 0.10 & $(0.43)$ \\
\hline GMOEx & $210.54,217.38,210.89,213.24$ & 1.02 & 0.16 & 0.09 & $(0.5)$ \\
\hline KwEx & $209.42,216.24,209.77,212.12$ & 0.74 & 0.11 & 0.09 & $(0.5)$ \\
\hline $\mathrm{BEx}$ & $207.37,214.21,207.73,210.09$ & 0.98 & 0.15 & 0.11 & $(0.34)$ \\
\hline MOKwEx & $209.44,218.56,210.04,213.04$ & 0.79 & 0.12 & 0.10 & $(0.44)$ \\
\hline KwMOEx & $207.82,216.94,208.42,211.42$ & 0.61 & 0.11 & 0.09 & $(0.5)$ \\
\hline BrXEx & $235.31,239.92,235.53,237.14$ & 2.92 & 0.52 & 0.22 & $(0.002)$ \\
\hline OIPGWEX & $206.88,215.98,207.47,210.50$ & 0.63 & 0.105 & 0.088 & $(0.639)$ \\
\hline
\end{tabular}

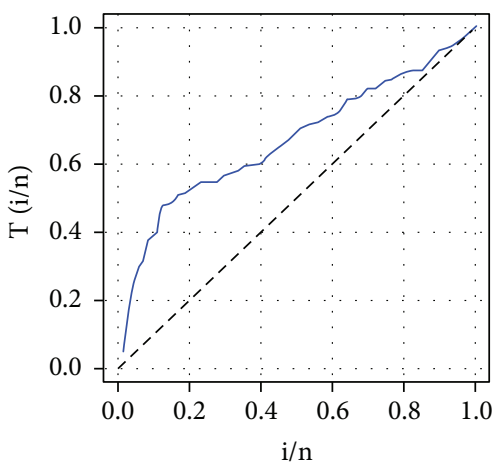

(a)

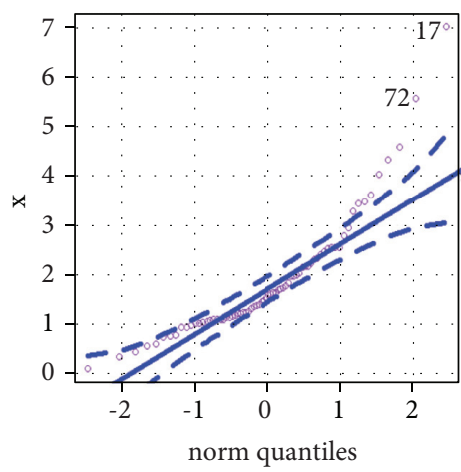

(b)

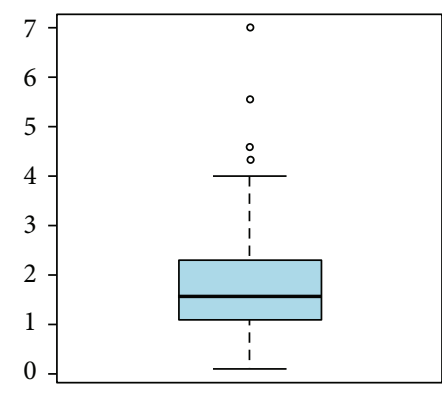

(c)

Figure 10: The TTT plot (a), Q-Q plot (b), and box plot (c) for the second data.

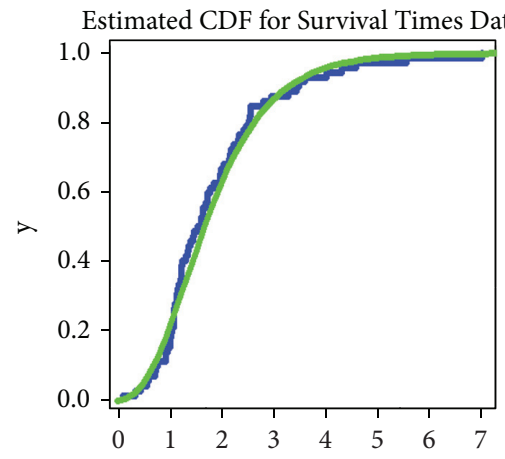

— The OIPGWE Distribution

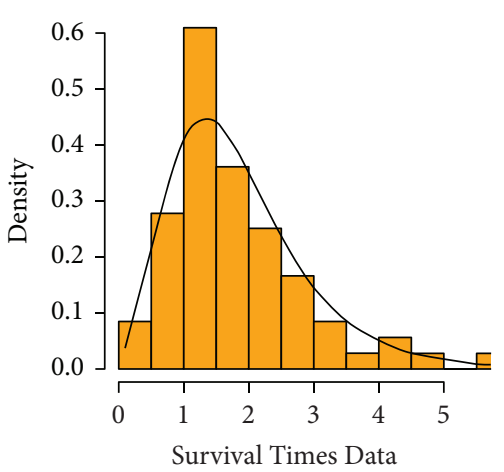

— The OIPGWE Distribution

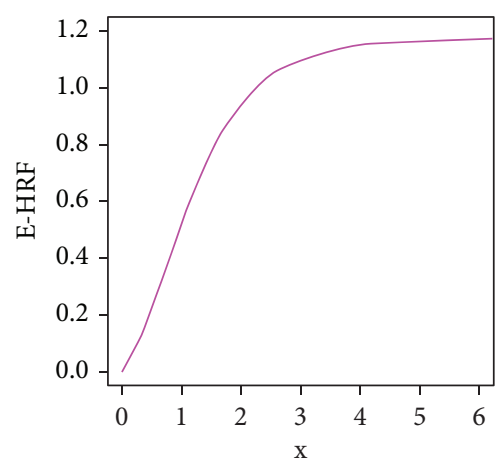

Figure 11: E-CDF, E-PDF, and E-HRF for second data. 

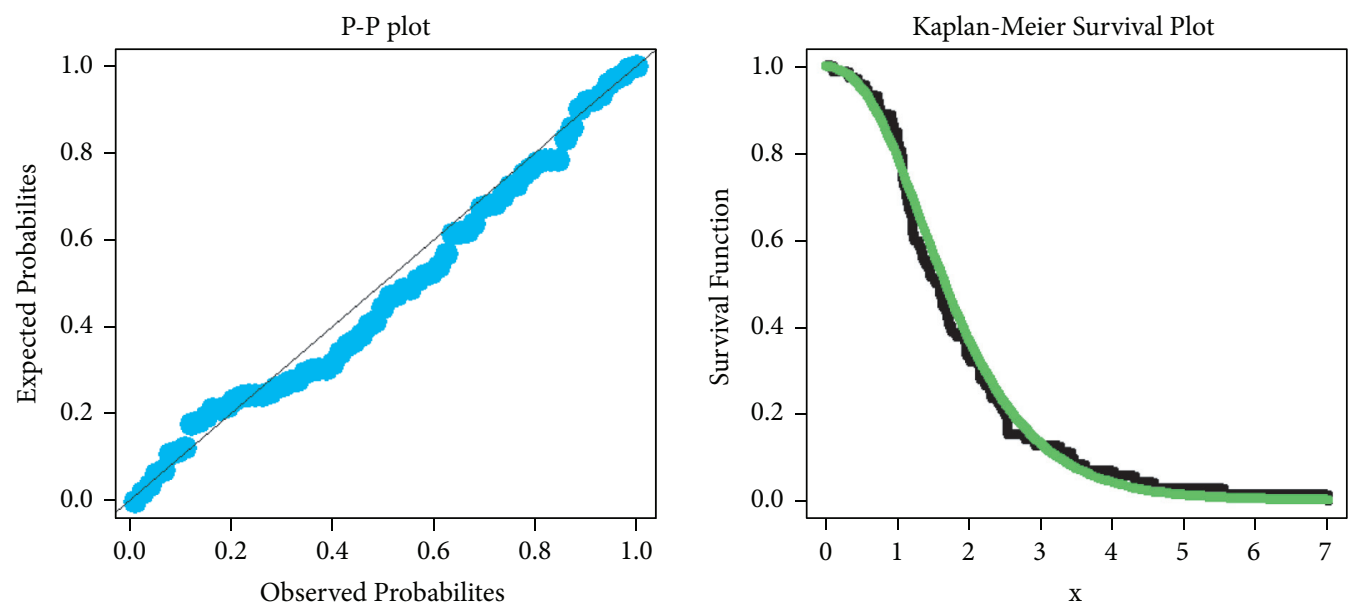

FIgURe 12: P-P plot and Kaplan-Meier survival plot for second data.

\section{Conclusions}

A novel flexibly generated family of distributions was developed and investigated in this study which is called OIPGW-G family. The new proposed family contains many new models, and its density can be right skewed and symmetric with unimodal and bimodal shapes. The new HRF of the new models can be "constant," "decreasing," "increasing," "increasing-constant," "upside-down-constant," and "decreasing-constant." Some of the mathematical properties of the new family are computed. Numerical calculations for the expected value, skewness, variance, and kurtosis are computed. Different types of entropies are calculated. Some numerical values of RéE, QE, HaChE, and ArE for some selected values of parameters for the OIPGWEx model are computed. Estimation of OIPGW-G parameters is performed by ML, LS, and WLS estimation methods. Some bivariate and multivariate OIPGWEx type models have been also derived. Two genuine data sets are used to demonstrate the family's utility and versatility. The OIPGWEx model is far superior to the Ex, OLEx, MOEx, MEx, LBHEx, GMOEx, BEx, MOKwEx, KwEx, BXEx, and KwMOEx models in modeling the two data sets according to the $C_{1}, C_{2}, C_{3}, C_{4}, A^{*}, C, K$. S., and P. V. statistics. In the future, we are planning to use this family to generate new statistical models and study its structural properties.

\section{Data Availability}

The data used in the study are included within the article.

\section{Conflicts of Interest}

The authors declare that there are no conflicts of interest with any organization or authors.

\section{References}

[1] G. Prakash, "Inverted exponential distribution under a bayesian viewpoint," Journal of Modern Applied Statistical Methods, vol. 11, no. 1, pp. 190-202, 2012.
[2] V. G. Voda, "On the inverse Rayleigh random variable," Rep Stat Appl Res, vol. 9, pp. 13-21, 1972.

[3] V. K. Sharma, S. K. Singh, U. Singh, and V. Agiwal, "The inverse Lindley distribution: a stress-strength reliability model with application to head and neck cancer data," Journal of Industrial and Production Engineering, vol. 32, no. 3, pp. 162-173, 2015.

[4] K. V. P. Barco, J. Mazucheli, and V. Janeiro, "The inverse power Lindley distribution," Communications in StatisticsSimulation and Computation, vol. 46, no. 8, pp. 6308-6323, 2017.

[5] A. Abd AL-Fattah, A. EL-Helbawy, and R. AL-Dayian, "Inverted Kumaraswamy distribution. Properties and estimation," Pakistan Journal of Statistics, vol. 33, no. 1, pp. 37-61, 2017.

[6] M. H. Tahir, G. M. Cordeiro, S. Ali, S. Dey, and A. Manzoor, "The inverted Nadarajah-Haghighi distribution: estimation methods and applications," Journal of Statistical Computation and Simulation, vol. 88, no. 14, pp. 2775-2798, 2018.

[7] A. S. Hassan, M. Elgarhy, and R. Ragab, "Statistical properties and estimation of inverted Topp-Leone distribution," Journal of Statistics Applications \& Probability, vol. 9, pp. 319-331, 2020.

[8] M. Selim, "Some theoretical and computational aspects of the inverse generalized power Weibull distribution," Journal of Data Science, vol. 17, no. 4, pp. 754-767, 2019.

[9] A. W. Marshall and I. Olkin, "A new method for adding a parameter to a family of distributions with applications to the exponential and Weibull families," Biometrika, vol. 84, pp. 641-652, 1997.

[10] N. Eugene, C. Lee, and F. Famoye, "Beta-normal distribution and its applications," Communications in Statistics-Theory and Methods, vol. 31, no. 4, pp. 497-512, 2002.

[11] A. Nascimento, K. F. Silva, M. Cordeiro, M. Alizadeh, H. Yousof, and G. Hamedani, "The odd Nadarajah-Haghighi family of distributions. Properties and applications," Studia Scientiarum Mathematicarum Hungarica, vol. 56, no. 2, pp. 1-26, 2019.

[12] F. Gomes, A. Percontini, E. de Brito, M. Ramos, R. Venancio, and G. Cordeiro, "The odd Lindley- G family of distributions," Austrian Journal of Statistics, vol. 46, no. 1, pp. 57-79, 2017.

[13] M. Haq and M. Elgarhy, "The odd Fréchet- G family of probability distributions," Journal of Statistics Applications \& Probability, vol. 7, no. 1, pp. 189-203, 2018. 
[14] M. H. Tahir, G. M. Cordeiro, M. Alizadeh, M. Mansoor, M. Zubair, and G. G. Hamedani, "The odd generalized exponential family of distributions with applications," Journal of Statistical Distributions and Applications, vol. 2, no. 1, pp. 1-28, 2015.

[15] M. A. Aldahlan, F. Jamal, C. Chesneau, I. Elbatal, and M. Elgarhy, "Exponentiated power generalized Weibull power series family of distributions: properties, estimation and applications," PLoS One, vol. 15, no. 3, pp. e0230004-e0230025, 2020.

[16] A. Zubair, M. Elgarhy, G. Hamedani, and N. Butt, "Odd generalized N-H generated family of distributions with application to exponential model," Pakistan Journal of Statistics and Operation Research, vol. 16, no. 1, pp. 53-71, 2020.

[17] A. Alzaatreh, C. Lee, and F. Famoye, "A new method for generating families of continuous distributions," Metron, vol. 71, no. 1, pp. 63-79, 2013.

[18] A. Rényi, "On measures of entropy and information," in Proceedings of the 4th Berkeley Symposium on Mathematical Statistics and Probability, Berkeley, CF, USA, June 1960.

[19] C. Tsallis, "Possible generalization of Boltzmann-Gibbs statistics," Journal of Statistical Physics, vol. 52, no. 1-2, pp. $479-487,1988$.

[20] J. Havrda and F. Charvat, "Quantification method of classification processes, concept of structural a-entropy," Kybernetika, vol. 3, no. 1, pp. 30-35, 1967.

[21] S. Arimoto, "Information-theoretical considerations on estimation problems," Information and Control, vol. 19, no. 3, pp. 181-194, 1971.

[22] D. Morgenstern, "Einfache beispiele zweidimensionaler verteilungen," Mitteilingsblatt fur Mathematische Statistik, vol. 8, pp. 234-235, 1956.

[23] D. J. G. Farlie, "The performance of some correlation coefficients for a general bivariate distribution," Biometrika, vol. 47, no. 3-4, pp. 307-323, 1960.

[24] E. J. Gumbel, "Bivariate exponential distributions," Journal of the American Statistical Association, vol. 55, no. 292, pp. $698-707,1960$.

[25] E. J. Gumbel, "Bivariate logistic distributions," Journal of the American Statistical Association, vol. 56, no. 294, pp. 335-349, 1961.

[26] D. B. Pougaza and M. A. Djafari, "Maximum entropies copulas," in Proceedings of the 30th International Workshop on Bayesian Inference and Maximum Entropy Methods in Science and Engineering, pp. 329-336, Boise, Idaho, March 2011.

[27] A. Al-babtain, A. Elbatal, I. Elbatal, and H. Yousof, A new flexible three-parameter model: properties, Clayton Copula, and modeling real data," Symmetry, vol. 12, no. 3, pp. 440-517, 2020.

[28] J. Gross and V. A. Clark, Survival Distributions: Reliability Applications in the Biometrical Sciences, John Wiley, New York, NY, USA, 1975.

[29] T. Bjerkedal, "Acquisition of resistance in Guinea pigs infected with different doses of virulent tubercle Bacilli1," American Journal of Epidemiology, vol. 72, no. 1, pp. 130-148, 1960. 